\title{
1 Activity-based urinary biomarkers of response and resistance to 2 checkpoint blockade immunotherapy
}

4 Authors: Quoc D. Mac ${ }^{1}$, Congmin $\mathrm{Xu}^{1}$, James R. Bowen ${ }^{1}$, Anirudh Sivakumar ${ }^{1}$, 5 Hathaichanok Phuengkham ${ }^{1}$, Fang-Yi Su${ }^{1}$, Samuel Z. Stentz ${ }^{1}$, Hyoungjun Sim ${ }^{1}$, Adrian 6 M. Harris ${ }^{1}$, Tonia T. Li ${ }^{1}$, Peng Qiu ${ }^{1,2,5,6}$, Gabriel A. Kwong ${ }^{1-6, *}$

Affiliations:

${ }^{1}$ Wallace H. Coulter Department of Biomedical Engineering, Georgia Tech College of Engineering and Emory School of Medicine, Atlanta, GA 30332, USA.

11 2Parker H. Petit Institute of Bioengineering and Bioscience, Atlanta, GA 30332, USA.

123 Institute for Electronics and Nanotechnology, Georgia Tech, Atlanta, GA 30332.

13 4Integrated Cancer Research Center, Georgia Tech, Atlanta, GA 30332.

$14{ }^{5}$ The Georgia Immunoengineering Consortium, Emory University and Georgia Tech, 15 Atlanta, GA 30332.

$16{ }^{6}$ Winship Cancer Institute, Emory University, Atlanta, GA 30322

17 *To whom correspondence should be addressed: gkwong@gatech.edu (G.A.K.)

INSIGHT

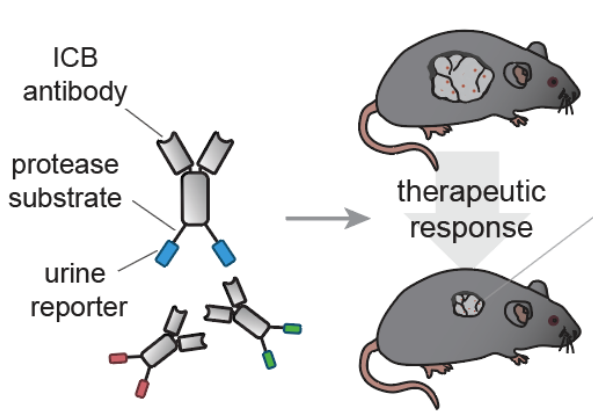

Anti-tumor immune activity

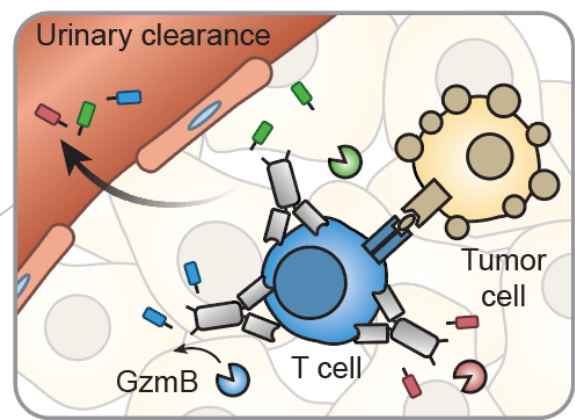

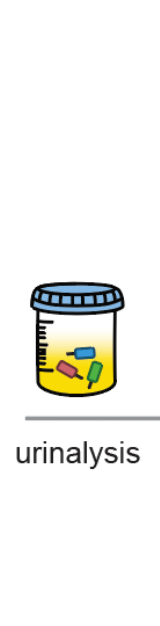

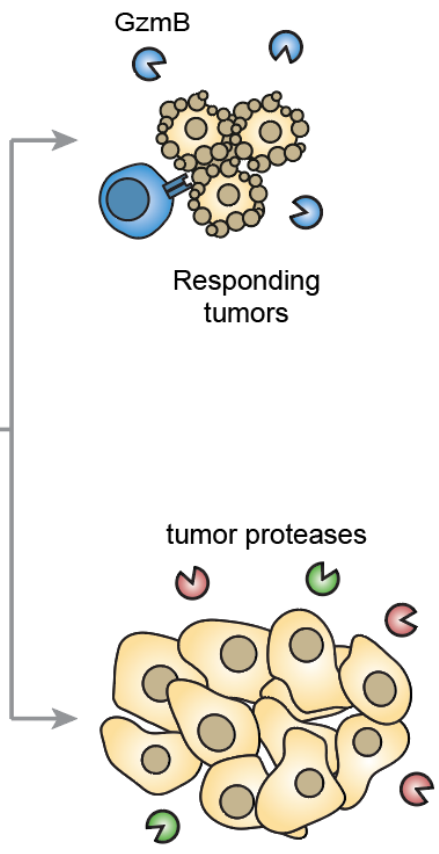

Nonresponding or resistant tumors (e.g., B2m LOF, Jak1 LOF) 


\section{Abstract}

Immune checkpoint blockade (ICB) therapy has transformed cancer treatment, yet

23 most patients do not derive clinical benefit and responders can acquire resistance to

24 therapy. Noninvasive biomarkers are needed to indicate early on-treatment response and

25 resistance mechanisms. Here we developed ImmuNe Sensors for monltorinG checkpoint

26 blockade Iherapy (INSIGHT), which comprises a library of mass-barcoded peptide

27 substrates conjugated to aPD1 antibodies, as therapeutic sensors. Following systemic

28 administration, INSIGHT carries out the dual role of reinvigorating $\mathrm{T}$ cell function and

29 profiling $\mathrm{T}$ cell and tumor proteases by the release of cleaved peptides into urine for

30 noninvasive detection by mass spectrometry. We show that an aPD1 therapeutic sensor

31 for Granzyme B discriminates early treatment responses before tumor volumes

32 significantly diverge from isotype controls in murine models of colorectal cancer. To

33 differentiate mechanisms of resistance by multivariate analysis, we design a mass-

34 barcoded, 14-plex INSIGHT library to profile proteases differentially expressed by tumors

35 harboring B2m or Jak1 loss-of-function mutations. We find that binary classifiers trained

36 on urine samples indicate response to aPD-1 therapy as early as the start of the second

37 dose, and discriminate $\mathrm{B} 2 \mathrm{~m}$ from Jak1 resistance with high sensitivity and specificity

38 (AUROCs > 0.9). Our data supports the use of activity-based biomarkers for early on-

39 treatment response assessment and classification of refractory tumors based on

40 resistance mechanisms. 


\section{Introduction}

Immune checkpoint blockade (ICB) therapy has transformed the treatment of cancer for patients across a broad range of malignancies ${ }^{1,2}$. ICB involves the

47 administration of antibodies that block inhibitory checkpoint molecules, such as the

48 cytotoxic T lymphocyte-associated protein 4 (CTLA-4) or the programmed cell death

49 protein 1 (PD-1), to reinvigorate an anti-tumor T cell response. Despite the potential for

50 ICB to produce durable clinical outcomes, a large fraction of patients do not derive clinical

51 benefit ${ }^{1,3}$. Objective response rates remain below $\sim 25 \%$ in many cancer types, largely

52 due to immunosuppressive factors in the tumor microenvironment (TME) (e.g., Tregs or

53 MDSCs) and primary tumor-intrinsic mutations ${ }^{1}$. In addition, responsive tumors can

54 acquire resistance during therapy such as in metastatic melanoma where up to one-third

55 of patients with initial responses to ICB therapy eventually relapse ${ }^{3}$. Both primary and

56 acquired resistance are driven by mechanisms that enable tumor cells to evade anti-

57 tumor immune responses, including defects in antigen presentation or IFNy response

58 pathway ${ }^{3,4}$. Therefore, developing noninvasive biomarkers of immune response and

59 resistance to ICB has emerged as a clinical priority ${ }^{5}$.

Patient responses to ICB therapy are currently assessed using a combination of

61 radiographic, tumor, and serum biomarkers ${ }^{5}$. Radiographic evaluation by RECIST criteria

62 is the standard assessment method and occurs after the first cycle of ICB therapy, which

63 consists of 3-4 doses administered within an 8-12-week window ${ }^{6-8}$. The observation of

64 atypical patterns of response to ICB has motivated continual refinement to the timing and

65 frequency of radiographic assessment such as the development of immune-related

66 response criteria (e.g., irRC, irRECIST) to account for phenomenon like 
67 pseudoprogression ${ }^{5,9}$. Tumor biomarkers such as PD-L1 expression have been shown to

68 enrich for populations with clinical benefit, but have limitations as predictive biomarkers

69 as at least $\sim 40-50 \%$ patient tumors with PD-L1 positivity do not experience objective

70 responses ${ }^{5,10}$. Other tumor biomarker strategies, such as assessing on-treatment

71 changes in tumor mutational burden (TMB) by whole exome sequencing ${ }^{11}$, are promising

72 and have been found to correlate with aPD1 response. However, these approaches

73 require serial biopsies, which in practice are not typically collected over the course of

74 therapy with attendant patient risks. Therefore, considerable interest is focused on

75 identifying noninvasive biomarkers to allow longitudinal and quantitative assessment.

76 These include quantifying changes in T cell clonality or circulating tumor DNA (ctDNA)

77 levels, which have been shown to be detectable within 3-4 weeks of treatment and

78 correlate with objective response and overall survival ${ }^{12-14}$. These studies highlight the

79 considerable interest and need for noninvasive and longitudinal assessment strategies to

80 track response and resistance to ICB therapy early on-treatment.

Proteases play fundamental roles in cancer biology, immunity, and anti-tumor

82 responses and therefore may provide a new mechanism to evaluate ICB therapy. Tumor-

83 dysregulated proteases (e.g., matrix metalloproteases, cathepsins) are involved in

84 proteolytic cascades that modify the tumor microenvironment (TME) during angiogenesis,

85 growth, and metastasis ${ }^{15,16}$. In addition, T cell-mediated tumor control is primarily carried

86 out by granzymes, which are serine proteases, released by cytotoxic $\mathrm{T}$ cells ${ }^{17}$. The

87 ubiquity of protease dysregulation has motivated the development of molecular imaging

88 probes for visualizing tumor or T cell proteases ${ }^{18-21}$, as well as synthetic biomarkers for

89 multiplexed quantification of protease activity from urine $22-27$. Building on these studies, 
90 we developed Immune Sensors for monltorinG cHeckpoint blockade Therapy (INSIGHT)

91 to detect tumor and immune proteases during treatment as activity-based biomarkers of

92 response and resistance. INSIGHT immune sensors consist of mass-barcoded protease

93 substrates conjugated to ICB antibodies that during the course of treatment are cleaved

94 by proteases, triggering the release of reporters that filter into urine. After urine collection,

95 cleaved reporters are quantified by mass spectrometry according to their mass barcode.

96 In preclinical animal models, we show that binary classifiers trained on protease

97 signatures by machine learning indicate on-treatment responses as early as the start of

98 the second dose and differentiate $\mathrm{B} 2 \mathrm{~m}$ and Jak1 resistance with high sensitivity and 99 specificity.

101 Results

102 Antibody-peptide therapeutic sensors retain target binding and in vivo therapeutic efficacy We first characterized target binding and therapeutic efficacy of ICB antibody-

104 peptide conjugates. As a representative formulation, we coupled a fluorescently labeled 105 peptide substrate selective for murine GzmB (IEFDSG ${ }^{26}$ ) to aPD1 (clone 8H3) to form an 106 aPD1-GzmB sensor conjugate (aPD1-GS) (Fig. 1a). To determine whether peptide 107 conjugation would interfere with PD1 binding, we tested different peptide:antibody 108 stoichiometric ratios $(0,1,3,5,7)$ and quantified binding to recombinant PD1 by ELISA. 109 We observed negligible differences in EC50 at a 1:1 ratio compared to unmodified aPD1

110 (3.6 vs. $2.1 \mathrm{nM}$ respectively) (Fig. 1b) but at higher ratios, a gradual reduction in binding 111 (up to $24 \mathrm{nM}$ at a 7:1 ratio) (SFig. 1a). To confirm that these results were not clone 112 dependent, we coupled GzmB peptides to another aPD1 clone (29F.1A12) at a 1:1 ratio 
113 and found that target binding was likewise preserved between aPD1-GS and

114 unconjugated antibody (EC50 = $0.15 \mathrm{nM}$ vs. $0.18 \mathrm{nM})$ (Fig. 1c). Based on these results,

115 we used a 1:1 conjugation ratio for all subsequent studies.

116 We next evaluated target binding of aPD1-GS to tumor infiltrating lymphocytes

117 (TILs) isolated from MC38 tumors since ligand presentation of plate-bound recombinant

118 PD1 may differ from endogenous PD1 expressed by T cells. We used the MC38 colon

119 adenocarcinoma syngeneic tumor model because these cancer cells have a high

120 mutation burden, which has been shown to lead to an endogenous $\mathrm{T}$ cell infiltrate

121 following aPD1 monotherapy ${ }^{28}$. Flow cytometry analysis of CD8+ TILs stained with either

122 aPD1-GS or unmodified aPD1 showed statistically equivalent PD1 expression by median

123 fluorescence intensity (MFI), indicating that peptide conjugation did not significantly affect

124 target binding to endogenous PD1 expressed on cell surfaces ( $\mathrm{n}=10$, Fig. 1d, e). We

125 further confirmed that peptide conjugation did not affect therapeutic efficacy by comparing

126 anti-tumor responses. Following a treatment schedule that involved four doses of

127 antibody to C57BL/6 mice bearing MC38 tumors, we observed no statistical difference in

128 tumor burden in mice given aPD1-GS or unmodified aPD1. Both formulations resulted in

129 smaller tumors that were statistically significant compared to animals given IgG1 isotype

130 control $(P \leq 0.0001, n=6$, Fig. 1f). Taken together, these data demonstrate that coupling

131 peptides at a low molar ratio to aPD1 does not affect target binding or in vivo therapeutic 132 efficacy. 
We next tested the ability of aPD1-GS to monitor GzmB activity in a T cell killing assay. To quantify cleavage activity by fluorimetry, we coupled GzmB peptides containing

137 a fluorophore-quencher pair (5FAM-AIEFDSG-CPQ2) to aPD1. (Fig. 2a). We assessed

138 substrate specificity by incubating aPD1-GS with fresh mouse serum, tumor-associated

139 proteases (e.g., cathepsin B, MMP9), or coagulation and complement proteases (e.g.,

140 C1s, thrombin). While incubation with recombinant GzmB led to a rapid increase in

141 sample fluorescence, incubation with mouse serum or recombinant proteases did not

142 result in detectable increases in fluorescence that would indicate cross-cutting of our

143 sensors (Fig. 2b). To evaluate aPD1-GS activation in the context of a T cell killing assay,

144 we cocultured Pmel T cells with gp100-expressing B16 melanoma cells at increasing

145 effector to target cell ratios $(0,1,5,10)$ and verified statistically significant increases in

146 both supernatant GzmB by ELISA and target cell death by lactose dehydrogenase (LDH)

147 release $(\mathrm{n}=3$, Fig. $\mathbf{2 c}$, d). Under these co-culture conditions, we observed significant 148 increases in fluorescence only in cocultures incubated with aPD1-GS, but not in control

149 wells containing unmodified aPD1 antibody or aPD1 conjugated with a control peptide

150 substrate (5FAM-ALQRIYK-CPQ2) ( $\mathrm{n}=3$, Fig. 2e). We also did not observe aPD1-GS

151 activation in cocultures of OT1 T cells and B16 cancer cells, which do not express the

152 OVA antigen. ( $\mathrm{P} \leq 0.0001, \mathrm{n}=4$, Fig. $2 \mathrm{f}$ ). Collectively, these data demonstrate that $\alpha \mathrm{PD} 1-$

153 GS is selectively cleaved by GzmB and can be used to detect T cell killing of tumor cells.

155 Noninvasive detection of early on-treatment response to ICB therapy

156 We next evaluated the potential of aPD1-GS to noninvasively detect response to

157 treatment in mouse models based on GzmB activity alone. Because free peptides can be 
158 rapidly degraded in blood but have improved pharmacokinetic profiles when conjugated

159 to an antibody or protein scaffold 29,30 , we first quantified the plasma concentration of

160 uncleaved aPD1-GS following intravenous administration to determine peptide stability.

161 We developed an indirect ELISA that uses plate-bound PD1 to capture aPD1-GS and a

162 detection antibody specific for the FAM reporter at the termini of the peptide substrate to

163 differentiate between cleaved and uncleaved conjugates (SFig. 2a). In validation assays,

164 we compared ELISA signals from samples that contained aPD1-GS with or without

165 preincubation with recombinant GzmB. Whereas aPD1-GS was readily detected

166 compared to unmodified aPD1, we observed dose dependent reduction in signals for

167 aPD1-GS samples treated with GzmB ( $\mathrm{n}=3$, SFig. $\mathbf{2 b}, \mathbf{2 c}$ ), validating the ability to

168 discriminate between cleaved and uncleaved conjugates. Using this assay, we

169 determined that the circulation half-life of uncleaved aPD1-GS was several hours and

170 statistically equivalent to unmodified $\alpha$ PD1 antibody $(3.9 \pm 1.3 \mathrm{~h}$ vs $6.5 \pm 4.2 \mathrm{~h}, \mathrm{n}=3$, two-

171 way ANOVA) (Fig. 3a), indicating peptide stability in circulation.

172 We evaluated aPD1-GS to detect response in C57BL/6 mice bearing MC38

173 tumors. We confirmed significantly elevated expression of GzmB in CD8+ TILs following

174 two doses of aPD1-GS compared to control mice that received an isotype antibody

175 conjugated with the same peptide (Iso-GS) $(P \leq 0.001, n=9$, Fig. 3b, c). To evaluate the

176 potential for serial on-treatment response assessment, we quantified the concentration of

177 cleaved fluorescent reporters in urine samples that were collected within 3 hours after

178 each dose was administered (day 7, 10, 14) (Fig. 3d). At the start of the first dose on day

179 7, urine signals from both cohorts of mice were statistically identical as expected. By

180 contrast, urine signals were significantly elevated in mice treated with aPD1-GS at the 
181 start of the second dose on day $10(P \leq 0.01, n=6-7)$ when tumors were statistically

182 equivalent in volume compared to control mice that received Iso-GS (255 $\mathrm{mm}^{3}$ vs. 441

$\left.183 \mathrm{~mm}^{3}, \mathrm{P}=0.68, \mathrm{n}=6-7\right)$. This difference in urine signals was further accentuated by the

184 start of the third dose on day $14(P \leq 0.0001, n=6-7)$ (Fig. 3e). Receiver operator

185 characteristic (ROC) analysis of reporter levels in urine samples revealed an area under

186 curve (AUC) of 0.86 and 1.00 for dose 2 and 3 respectively (Fig. 3f), indicating the ability

187 to differentiate ICB response with high sensitivity and specificity.

We further sought to confirm urinary detection in a different preclinical model using

189 BALB/c mice bearing syngeneic CT26 tumors that respond to combination therapy (aPD1

190 and aCTLA4) but minimally to monotherapy (aPD1 or aCTLA4) 31,32 . Compared to

191 matched isotype control conjugates, monotherapy with either aPD1-GS or aCTLA4-GS

192 did not result in statistical differences in tumor burden and urine signals across all doses

193 (SFig. 3a, b, c, d). By contrast, combination treatment with aPD1-GS and aCTLA4

194 resulted in significantly lower tumor burden $(P \leq 0.0001, n=7-14$, Fig. $3 g$, higher levels

195 of GzmB+CD8+ TILs ( $P \leq 0.05, n=7$, SFig. 4a, b), and significant increases in urine

196 signals at the start of the second or third dose (AUROC $=0.95$ and 0.92 respectively, Fig.

197 3h). Similar to results observed in the MC38 study, urine analysis indicated response to

198 treatment several days before tumor volumes were statistically different compared to

199 control mice (day 14 vs 17$)(P \leq 0.0001, n=7-14$, Fig. 3i). Collectively, these results

200 showed that aPD1-GS indicated response to ICB treatment as early as the start of the

201 second dose with high sensitivity and specificity. 
Tumor resistance mechanisms to ICB include loss-of-function (LOF) mutations in $\mathrm{B} 2 \mathrm{M}$, a protein subunit of $\mathrm{MHC}-\mathrm{I}$, and JAK1, an essential signaling protein of the IFNy response pathway ${ }^{3,4}$. To model resistance, we knocked out (KO) B2m or Jak1 from wildtype (WT) MC38 tumor cells with CRISPR/Cas9. We validated KO cells by TIDE (Tracking of Indels by Decomposition) analysis ${ }^{33}$ (SFig. 5a), loss of surface expression

209 of $\mathrm{MHC} \mathrm{I}(\mathrm{H} 2-\mathrm{Kb})$ in B2m-1- cells by flow cytometry (SFig. 5b), reduction in GzmB and 210 IFNy expression by OT1 T cells after co-culture with OVA-pulsed B2m ${ }^{-/-}$MC38 target

211 cells $(P \leq 0.05, n=3$, SFig. 5c), and lack of upregulation of H2-Kb and PD-L1 following

212 IFNy stimulation of Jak1-/- cells (SFig. 5d). To confirm resistance to ICB therapy, we

213 treated mice bearing $\mathrm{WT}, \mathrm{B} 2 \mathrm{~m}^{-/-}$, or Jak1 ${ }^{-/-} \mathrm{MC} 38$ tumors with either aPD1 or IgG1

214 isotype control. Whereas aPD1 treatment of WT tumors resulted in significantly smaller

215 tumors and improved survival $(\mathrm{MST}=30)$ relative to isotype control $(\mathrm{MST}=21)(\mathrm{P} \leq$

216 0.0001, $n=25$, Fig. 4a, SFig. 6), no statistical differences in tumor burden and overall

217 survival were observed in mice with $\mathrm{B} 2 \mathrm{~m}^{-/-}$or Jak1-/- tumors. Together, our data

218 confirmed that LOF mutations in B2m and Jak1 render MC38 tumors resistant to aPD1

219 therapy.

To quantify the breadth of protease dysregulation in ICB response and resistance,

221 we sequenced the transcriptomes of WT, B2m $\mathrm{m}^{--}$, and Jak1 $1^{-/-}$MC38 tumors after two 222 doses of either aPD1 or IgG1 ( $n=5)$. By t-Distributed Stochastic Neighbor Embedding (t223 SNE) analysis, we observed three distinct gene clusters corresponding to WT, B2m-,

224 and Jak1 ${ }^{-/}$tumors (Fig. 4b). Gene set enrichment analyses (GSEA) ${ }^{34}$ confirmed 225 enrichment of immune pathways (e.g., IFNy response, IL2-STAT5 signaling, 226 inflammatory response, complement) in WT tumors in response to PD1 therapy, with 
227 minimal enrichment or downregulation in $\mathrm{B} 2 \mathrm{~m}^{-/-}$and Jak1 ${ }^{-/-}$tumors, respectively $(\mathrm{P} \leq$

228 0.05, Fig. 4c, SFig. 7a). To compare with patient ICB responses, we performed GSEA

229 on bulk tumor RNA-Seq data from advanced melanoma patients treated with aPD1

230 monotherapy ${ }^{11}$ that were classified into complete or partial responders $(C R+P R)$,

231 progressive disease (PD), or stable disease (SD) based on RECIST criteria ${ }^{35}$. We

232 observed enrichment in immune pathways that were similar to murine tumors (e.g., IFNy

233 response, IL2-STAT5 signaling, complement) in CR + PR relative to PD (P $\leq 0.05$, Fig.

234 4c, SFig. 7b).

235 To identify proteases dysregulated in ICB response and resistance, we compared

236 RNA transcripts levels of WT tumors on aPD1 or IgG1 treatment and observed that the

237 top differentially expressed proteases, as selected by a log2 fold change threshold greater

238 than 1, were from the granzyme, metalloproteinase, and cathepsin family of enzymes ( $P$

$239 \leq 0.05$, Fig. 4d, SFig. 8a). By comparison, B2m-1- tumors on aPD1 treatment showed

240 broader dysregulation that included proteases from the complement, coagulation, and

241 caspase families compared to Jak1 $1^{-1-}$ tumors (log2 fold change $>1, P \leq 0.05$, Fig. 4e,

242 SFig. 8b). Similar to our mouse models, human melanoma tumors in patients ${ }^{11}$ that had

243 a complete or partial response to ICB were characterized by significant upregulation of

$244 \sim 20$ proteases across the same protease families relative to progressive disease (log2

245 fold change $>1, P \leq 0.01$, Fig. 4f). By unsupervised hierarchical clustering, protease

246 expression profiles were primarily grouped into CR+PR compared to PD (SFig. 8c).

247 Taken together, these data indicate that proteases are differentially regulated during

248 response and resistance to ICB therapies. 
Multiplexed detection of protease activity by mass spectrometry.

We next designed substrates for our INSIGHT library to detect the proteases

252 differentially expressed in ICB response and resistance (Fig. 5a). We compiled published

253 substrate sequences for five target protease families - granzymes, metalloproteases,

254 coagulation and complement proteases, caspases, and cathepsins - and synthesized a

255 candidate library of 66 fluorogenic substrates, which consisted of 6-11 amino acids

256 flanked by a fluorophore (FAM) and a quencher (Dabcyl). We tested each substrate

257 against 17 recombinant proteases (2+ per family) and quantified cleavage efficiency

258 based on the fold change in fluorescence at 60 minutes (Fig. 5b, SFig. 9). To facilitate

259 downselection, we applied t-SNE analysis and observed 4 major substrate clusters:

260 cluster 1 contained substrates preferentially cleaved by metalloproteases, cluster 2 by

261 metalloproteases and cathepsins, cluster 3 by coagulation and complement proteases,

262 and cluster 4 by granzymes and caspases (Fig. 5c). From each cluster, we selected 3 or

263 more representative substrates to form a final library of 14 substrates. Each substrate in

264 this set was characterized by a 2-22 fold increase in fluorescence in the presence of

265 target proteases (Fig. 5d), and the majority of substrate pairs (76\%) had a Spearman's

266 correlation coefficient (Rs) less than 0.5, indicating low redundancy of the library (SFig.

267 10).

To enable multiplexed detection by mass spectrometry, we designed 14 mass

269 barcodes by enriching the peptide reporter glutamate-fibrinopeptide B (Glufib)

270 (EGVNDNEEGFFSAR) with different distributions of stable isotopes. As described

271 previously ${ }^{22}$, this approach allows multiple reporters that share the same MS1 parent

272 mass to be differentiated by unique quantifier MS2 fragments by tandem mass 
273 spectrometry (MS/MS) (Table 1). For validation, we derivatized our 14-plex substrate

274 library with mass barcodes and confirmed that MS2 signals were linearly correlated with

275 substrate concentrations $\left(R^{2} \geq 0.96\right.$, Fig. 5e) and that the mass barcoded substrates

276 conjugated to aPD1 or IgG1 antibody were quantifiable after cleavage ( $\mathrm{n}=3$, Fig. 5f).

277 Our results showed that INSIGHT substrates are sensitive to cleavage by dysregulated

278 proteases in the context of ICB response and resistance, and mass-barcoding allows

279 multiplexed quantification of substrates.

281 Binary classification of response and resistance by 14-plex INSIGHT

To assess the potential of our 14-plex INSIGHT library to detect early on-treatment

283 response to ICB therapy, we administered 14-plex aPD1 or IgG1 conjugates to mice

284 bearing WT MC38 tumors at days 7, 10, and 13 (Fig. 6a). At each timepoint, urine

285 samples were collected within three hours after intravenous administration and cleavage

286 fragments were quantified by mass spectrometry. Urinary signals from dose 2 and 3 were

287 normalized to dose 1 to account for pre-treatment baseline activity. We applied random

288 forest classification to the data split into training and test sets by 5 -fold cross validation

289 and repeated this procedure 100 times to obtain the average area under the ROC curve

$290(\mathrm{AUC})^{36}$. Under these conditions, INSIGHT discriminated aPD1-treated mice $(n=25)$ from

291 isotype controls $(n=15)$ with high accuracy (AUC $=0.92[95 \% \mathrm{Cl}=0.88-0.95]$, sensitivity

$292(\mathrm{Se})=87 \%$, specificity $(\mathrm{Sp})=86 \%$ ) as early as the start of the second dose, with

293 statistically identical classification performance at dose 3 (AUC $=0.93[0.90-0.95], P=$

294 0.650, paired Student's t-test) (Fig. 6b). To assess the relative weight of each probe, we

295 quantified the feature importance score and observed that probes L2-8, L3-7 and L2-1 
296 had the largest contribution to classification accuracy with aggregate scores for dose 2 297 and 3 above 0.6 compared to scores of 0.3 and below for all other probes (Fig. 6c). These 298 three probes were selective for granzymes, MMPs and cathepsins, including substrate 299 L2-1 which was the same sequence previously used in aPD1-GS (Fig. 3). Based on the marked difference in feature importance scores, we further tested whether L2-8, L3-7, 301 and L2-1 alone were sufficient to classify ICB responses, and found that the 3 probe set 302 classified response with AUCs greater than 0.9 for both doses (dose 2 AUC $=0.95$ [0.930.97]; dose 3 AUC $=0.91$ [0.87-0.93]) with no statistical reduction in accuracy compared 304 to the 14-plex panel $(P=0.147$ on dose $2, P=0.317$ on dose 3 , Fig. 6 d, SFig. 11). These 305 data indicated that INSIGHT discriminated ICB responders as early as the second dose 306 with 3 probes out of the 14-plex set.

We conducted similar longitudinal experiments to assess the ability of INSIGHT to 308 stratify refractory tumors based on B2m $\mathrm{m}^{-/-}(n=15)$ or Jak1 ${ }^{-/-}(n=15)$ LOF mutations (Fig.

309 6a). Following urine quantification by mass spectrometry, random forest classification 310 resulted in an AUC of $0.77(95 \% \mathrm{Cl}=0.71-0.82, \mathrm{Se}=84 \%, \mathrm{Sp}=65 \%)$ on dose 2 , which 311 significantly increased to $0.91(95 \% \mathrm{Cl}=0.86-0.94, \mathrm{Se}=87 \%, \mathrm{Sp}=81 \% ; \mathrm{P} \leq 0.0001)$ on 312 dose 3 (Fig. 6e). By feature importance analysis, we observed that a larger number of 313 probes contributed to resistance classification where the top 5 probes had aggregate 314 scores above 0.45 while the previous top ICB response probes, L2-8, L3-7 and L2-1, 315 were in the bottom half by rank order (Fig. 6f). We further asked whether a minimal probe 316 set could stratify resistance and by iterative analysis, we found that the top 5 probes (L2317 11, L2-20, L2-19, L3-16, and L2-9) classified B2m ${ }^{-/-}$from Jak1 ${ }^{-/-}$resistance with 318 statistically equivalent performance to the full INSIGHT library (dose 2 AUC $=0.80[0.74-$ 
319 0.84], $P=0.430 ;$ dose 3 AUC = 0.91 [0.86-0.94], P > 0.999; Fig. 6d, SFig. 11). Given that

320 this subset of 5 probes did not contribute to the response monitoring classifier, we

321 compared the importance score for all 14 probes for both classification tasks and found

322 a strong negative correlation $(R=-0.896)$ between the top probes for response monitoring

323 (L2-1, L3-7, and L2-8) and stratifying resistance (L2-11, L2-20, L2-19, L3-16, and L2-9)

324 (Fig. 6g). Our data indicated that binary classifiers trained on INSIGHT measurements of 325 protease activity discriminate response and resistance to ICB therapies in mouse models.

\section{Discussion}

In light of the central role proteases play in T cell cytotoxicity and tumor biology,

329 our study focused on demonstrating INISIGHT as an activity-based platform to track early

330 response and resistance to ICB therapies. We showed that aPD1-peptide conjugates act

331 as therapeutic sensors that carry out the dual roles of reinvigorating $\mathrm{T}$ cell function and

332 reporting on treatment response by the release of protease-cleaved reporters into urine

333 for noninvasive detection. Our results with a single aPD1-GS probe to quantify GzmB

334 activity in vivo showed that urinalysis of cleavage fragments anticipated response as early

335 as the start of the second dose before tumor volumes began to diverge between treated

336 and untreated animals. By transcriptomic analysis, we identified proteases across five

337 families that were broadly dysregulated in tumors harboring $\mathrm{B} 2 \mathrm{~m}^{-/-}$or Jak1 ${ }^{-/-} \mathrm{LOF}^{-}$

338 mutations. This list of proteases formed the basis of a bespoke 14-plex INSIGHT library

339 that allowed binary classifiers trained on urine samples by machine learning to stratify the

340 mechanism of resistance with high diagnostic accuracy. Our results support the 
341 development of activity-based biomarkers for noninvasive and longitudinal assessment

342 of response and resistance to ICB therapies.

GzmB is the most potent pro-apoptotic granzyme and its release from granules

344 accompanied by perforin is a primary mechanism by which CD8+ T cells exert tumoricidal

345 activity. Compared to other tumor biomarkers (e.g., PD-L1 ${ }^{37}, \mathrm{TMB}^{38}, \mathrm{~T}$ cell-inflamed gene

346 expression profile $(\mathrm{GEP})^{39}$, microsatellite instability $(\mathrm{MSI})^{40}$ ) and serum biomarkers (e.g.,

347 ctDNA ${ }^{14,41}$, TCR clonality ${ }^{12,13}$, memory phenotypes ${ }^{12,13,42}$ ) under investigation, GzmB is a

348 direct biomarker of $\mathrm{T}$ cell cytotoxicity, and its expression has been shown to be

349 significantly upregulated in patient tumors responsive to aPD1 and aCTLA4 therapies ${ }^{43-}$

35045 . GzmB expression, therefore, has potential as an early biomarker of ICB response.

351 Recent work on a peptide PET probe that irreversibly binds to $\mathrm{GzmB}^{21,46}$ demonstrated

352 that high GzmB signals predicted early response to checkpoint therapy before changes

353 in tumor volumes were apparent in animal models. Similarly, we observed that tumor

354 treatment with aPD1-GS therapeutic sensors led to quantifiable levels of cleaved peptides

355 in urine that anticipated responders from isotype controls before tumor volumes

356 significantly diverged. As our peptide sensors are conjugated to therapeutic antibodies

357 and administered at the time of treatment, a separate infusion of diagnostic agents is not

358 required and response assessment can occur several hours after urine collection. In

359 longitudinal studies with mice treated with multiple doses, we observed changes by

360 urinalysis that indicated response as early as the start of the second dose of treatment.

361 GzmB expression by itself, however, is not a specific biomarker of ICB response

362 but rather a general biomarker of $\mathrm{T}$ and NK cell cytotoxicity that could be elevated under

363 confounding conditions such as reactivation of latent viruses or opportunistic infections ${ }^{47-}$ 
364 49. Moreover, a univariate GzmB biomarker also lacks the ability to differentiate

365 mechanisms of resistance that similarly result in loss of T cell cytotoxicity. Therefore, we

366 investigated whether a multiplexed INSIGHT library could provide the ability to assess

367 response and resistance to ICB therapy by multivariate classification. By transcriptomic

368 analysis, we found that proteases are broadly dysregulated across multiple enzyme

369 families both in tumors that respond to therapy and in tumors that harbor LOF mutations

370 in B2M or JAK1 genes that underpin resistance to checkpoint inhibitors ${ }^{3,4}$. These

371 proteases informed the design and selection of a 14-plex INSIGHT library that broadly

372 covered protease cleavage space to provide the ability to generate high-dimensional data

373 by mass spectrometry for classifier training. We observed that although the same

374 INSIGHT library was used in our animal studies, separate subsets of 3 to 5 probes were

375 ranked highest in importance depending on whether the use case was response

376 monitoring (L2-1, L3-7, and L2-8) or stratifying resistance mechanisms (L2-11, L2-20, L2-

377 19, L3-16, and L2-9). These probes were strongly anti-correlated $(R \sim-0.9)$ and binary

378 classifiers that were trained only on these minimal probe sets recapitulated the diagnostic

379 performance of the entire 14-plex library without reductions in classification accuracy

380 (AUROCs > 0.90). These observations lend support for a potential future strategy for

381 human testing that involves using the same superset of probes to train separate

382 classifiers for each intended use case. Following classifier validation, a down-selection

383 process could then be employed to reduce the number of probes to a minimal set. This

384 strategy may ensure the ability to generate high-dimensional data while reducing

385 regulatory burden associated with the need to test the safety and immunogenicity of

386 separate probe compositions. 
Several key areas warrant future study. Our work outlined a general development pipeline for activity-based biomarkers that involves nomination of candidate proteases based on established biology or transcriptomic analysis, substrate design and selection, 390 and classifier training and validation. Transcriptomic analysis of a large set of resistant 391 tumors (i.e., primary, adaptive and acquired) with different mechanisms of action (e.g., 392 absence of antigen presentation, insensitivity to T cells, genetic T cell exclusion ${ }^{3}$ ) would 393 further serve to nominate differentially expressed proteases and determine the extent of 394 conservation across cancer types and ICB therapies (e.g., aPD1 versus aCTLA-4). Given 395 that proteases that are closely related cleave similar substrates such as the $\mathrm{MMPs}^{50}$, cathepsins ${ }^{51}$ and caspases $^{52}$, our peptide selection process did not exclude substrates

397 with broad selectivity for proteases within a family, which is a challenge shared by the 398 field. This implies that assigning protease specificity to the cleavage signals will be 399 challenging without developing probes with exquisite selectivity for target proteases, 400 which may be possible with non-natural amino acids ${ }^{53,54}$, or mathematical algorithms to 401 deconvolve complex protease signatures ${ }^{55,56}$. Looking forward, phase 1 studies are 402 necessary to establish the safety of aPD1-peptide conjugates, which we anticipate to be 403 well-tolerated in humans given its composition is similar to protease-activatable masked 404 antibodies $^{29}$ and $T$ cell engagers ${ }^{57}$ that are undergoing clinical efficacy studies. The 405 classifiers we described in this study are relevant for the mouse models and should not 406 be directly mapped to humans without conducting separate training and validation 407 studies. Overall, our results support INSIGHT as an activity-based biomarker platform to 408 noninvasively track early response and resistance to ICB therapies from urine. 
Materials and Methods

\section{Animals}

412 6- to 8-week old female mice were used at the outsets of all experiments. Pmel (B6.Cg413 Thy1a/Cy Tg(TcraTcrb)8Rest/J ) and OT1 (C57BL/6-Tg(TcraTcrb)1100Mjb/J) transgenic 414 mice were bred in house using breeding pairs purchased from Jackson Lab. C57BL/6 415 and BALB/c mice for tumor studies were purchased from Jackson Lab. All animal 416 procedures were approved by Georgia Tech IACUC (protocol \#KWONG-A100193).

\section{Antibody-peptide conjugation.}

418 FITC-labelled GzmB substrate peptides ((FITC)AIEFDSGc; lower case letters = d-form amino acids) were synthesized by Tufts University Core Facility and used for in vivo 420 formulations. FITC-labelled GzmB substrate peptides with internal quencher ((5421 FAM)alEFDSGK(CPQ2)kkc) were synthesized by CPC Scientific and used for all in vitro 422 activity assays. Peptides with isobaric mass repoters were synthesized in housed using the Liberty Blue Peptide Synthesizer (CEM). Free aPD1 (kind gift of Dr. Gordon Freeman, Dana-Farber) and aCTLA4 (BioXCell; clone 9H10) antibodies were first reacted to the heterobifunctional crosslinker Succinimidyl lodoacetate (SIA; Thermo, 5:1 molar ratio) for 2 hours at room temperature (RT) in the dark, and excess SIA were removed by buffer exchange using Amicon spin filter (30 kDa, Millipore). Cysteine-terminated peptides were mixed with mAb-SIA (10:1 molar ratio) and reacted overnight at RT in the dark to obtain mAb-peptide conjugate. The conjugates were purified on a Superdex 200 Increase 10300 GL column using AKTA Pure FPLC System (GE Health Care). Endotoxin was removed from the samples by phase separation with Triton X-114 (Sigma) at $2 \%$ final volume ratio ${ }^{58}$. Final endotoxin concentrations were quantified by Pierce LAL Chromogenic Endotoxin Assay Kit (Thermo). Protein concentrations were determined by Pierce Protein Assay Kit (Thermo). Conjugates were buffered exchanged into PBS and sterile filtered before in vivo usage. Conjugation ratios of fluorescently labeled peptides were determined by corrected absorbance measurements by NanoDrop (Thermo). Conjugation of mass-encoded peptides were validated by MALDI using Autoflex mass spectrometer (Bruker).

PD-1 binding.

440 Binding of aPD1 conjugates to recombinant PD1 ligand was quantified using an ELISA assay developed in house, in which a high protein binding plate was coated with $1 \mathrm{ug} / \mathrm{mL}$ of recombinant Mouse PD-1 Protein (R\&D, 9047-PD-100). Binding of intact aPD1-GS conjugates was quantified in a sandwich ELISA using the same PD-1 coated plate. After sample incubation, $\alpha F I T C$ mAb (Thermo, 13-7691-82; 1:800 dilution staining concentration) was used for secondary staining. ELISA development was performed according to well-established protocol ${ }^{59}$. 
For half-life characterization, unconjugated aPD1 or aPD1-GS (100 ug) was administered i.v. to naïve C57BL/6 mice (Jackson Labs). At several time points following administration, blood was collected into Capillary Tubes (VWR), and serum was isolated by centrifugation. Serum concentrations of unconjugated aPD1 and aPD1-GS were determined by the PD1 binding and intact PD1 ELISA respectively.

aPD1 was conjugated with GzmB peptide substrates carrying an internal CPQ2 quencher to allow cleavage detection by fluorescent measurements. aPD1-GS (1.3 uM by peptide) was incubated in PBS at $37^{\circ} \mathrm{C}$ with fresh mouse serum, murine Granzyme B $(0.17 \mu \mathrm{M}$; Peprotech), human thrombin (13.5 $\mu \mathrm{M}$; HaemTech), mouse thrombin (12.5 $\mu \mathrm{M}$; HaemTech), cathepsin B (1.5 $\mu \mathrm{M}, \mathrm{R} \& \mathrm{D}), \mathrm{C} 1 \mathrm{r}$ (1.43 $\mu \mathrm{M}$; Sigma), C1s (1.80 $\mu \mathrm{M}$; Sigma), MMP9 (0.1 $\mu \mathrm{M}, \mathrm{R} \& \mathrm{D})$. Sample fluorescence was measured for 60 minutes using Cytation 5 plate reader (Biotek).

\section{Sensing protease activity during $T$ cell killing}

B16-F10 cells (ATCC) were cultured in DMEM supplemented with 10\% FBS and 1\% penicillin-streptomycin (Thermo). CD8+ T cells were isolated from either OT1 or Pmel (Jackson Labs) splenocytes by MACS using CD8a Microbeads (Miltenyi). Cells were activated by seeding in 96 -well plates pre-coated with anti-mouse CD3e $(1 \mu \mathrm{g} / \mathrm{ml}$ working concentration, Clone: 145-2C11, BD) and anti-mouse CD28 (2 $\mu \mathrm{g} / \mathrm{ml}$ working concentration, Clone: 37.51 , BD) at $2 \times 10^{6}$ cells $/ \mathrm{ml}$ in RPMI 1640 supplemented with 10\% FBS, 100U/ml penicillin-streptomycin, 1X non-essential amino acids (Gibco), 1mM sodium pyruvate, 0.05mM 2-mercaptoethanol, and 30U/ml hIL-2 (Roche). After 2 days, cells were washed and transferred to untreated culture flasks for expansion. Between day 4 to 6 after activation, activated T cells were washed before coincubated with $3 \times 10^{4}$ B16 target cells at various $T$ cell to effector cell ratios. After 48 hours, coculture supernatants were collected for LDH and GzmB measurements by the Pierce LDH Cytotoxicity Assay Kit (Thermo) and GzmB Mouse ELISA Kit (Thermo, BMS6029) respectively. To assess sensor activation during $T$ cell killing, cocultured of $T$ cells and target cells were spiked in with either aPD1-GS, aPD1 conjugated with control peptide (LQRIYK), and unconjugated aPD1. After 48 hours, fluorescence of coculture supernatant were measured using

\section{Tumor models}

CT26 (ATCC), MC38 (kind gift of the $\mathrm{NCl}$ and Dr. Dario Vignali, University of Pittsburgh), and $\mathrm{B} 2 \mathrm{~m}^{-/-}$vs. Jak1 ${ }^{-/-}$MC38 tumor cells were cultured in DMEM supplemented with $10 \%$ FBS and 1\% penicillin-streptomycin (Thermo). Cells were grown to a good density ( $70 \%$ confluence) before trypsinized for tumor inoculation. On the day of inoculation, C57BL/6 and BALB/C mice were shaved and injected s.c. into the left flank with either $1 \times 10^{6} \mathrm{MC} 38$ or CT26 cells respectively. Tumor burden were monitored until average tumor volume, quantified as $0.52 \times$ length $x$ width $x$ depth, was approximately $100 \mathrm{~mm}^{3}$ before initiating 
488

treatment. Mice were administered with aPD1 and/or aCTLA4 antibody-sensor conjugates or matched isotype control (100-150 ug/injection) every 3 or 4 days.

\section{Flow cytometry analysis of intratumoral $T$ cells}

Tumor dissociation and staining for flow cytometry. Less than $1 \mathrm{~g}$ of murine tumors were enzymatically and mechanically dissociated using Mouse Tumor Dissociation Kit (Miltenyi) and gentleMACS Dissociator (Miltenyi). TILs were then isolated from the single cell suspension using a density gradient with Percoll Centrifugation Media (GE Life Sciences) and DMEM Media (10\% FBS, 1\% Penstrep) at 44:56 volume ratio. TILs were counted with Trypan Blue (Thermo), and approximately $1 \times 10^{6}$ viable cells per sample were stained for flow cytometry analysis. Cells were first stained for surface markers in FACS Buffer (1x DPBS, 2\% FBS, 1 mM EDTA, 25 mM HEPES). Intracellular staining was performed using eBioscience Intracellular Fixation \& Permeabilization Buffer Set (Thermo). All antibodies were used for staining at 1:100 dilution from stock concentrations. Stained cells were analyzed by LSRFortessa Flow Cytometer (BD).

Antibody clones. CD45 (30-F11), CD8 (53-6.7), CD44 (IM7), PD-1 (29F.1A12), TIM3 (RMT3-23), CD4 (RM4-5), NK1.1 (PK136), CD19 (6D5), GZMB (GB12). Viability was accessed by staining with LIVE/DEAD Fixable Dye (Thermo).

\section{Urinary detection of therapeutic response and resistance to ICB therapy}

At 3 hours after administration of ICB antibody-sensor conjugates, urine was collected and analyzed for noninvasive detection of therapeutic response and resistance. FITC reporters were isolated from urine samples using Dynabeads (Thermo) decorated with aFITC antibody (Genetex). Sample fluorescence was measured by Cytation 5 plate reader (Biotek), and reporter concentrations were determined by using a known FITC ladder. Concentrations of isobaric mass reporters were quantified by Syneous Health (Morrisville, NC) using LC-MS/MS.

\section{Cas9 knockout of B2m and Jak1.}

CRISPR guide RNA's were designed to target two exons in either B2m ( $g 1$ : GACAAGCACCAGAAAGACCA, g2: GGATTTCAATGTGAGGCGGG) or Jak1 (g1: GTGAACTGGCATCAAGGAGT, g2: GCTTGGTGCTCTCATCGTAC) in the Mus musculus GRCm38 genome. Top and bottom guide oligonucleotides were annealed using T4 PNK (NEB) and ligated into the backbone of eSpCas9_PuroR_GFP plasmid (Sigma) using Bbsl cut sites and T7 ligase (NEB). $1 \times 10^{5}$ MC38 cells were transfected with gRNA-ligated eSpCas9 plasmids for 48 hours using TransIT-LT1 transfection reagent (Mirus Bio) in Opti-MEM (Thermo Fisher) and cultured for 3 passages in DMEM supplemented with 10\% FBS and 1\% penicillin-streptomycin (D10). Selection of transfected cells were done by supplementing culture media with $2 \mathrm{ug} / \mathrm{mL}$ puromycin (Thermo Fisher). Cells incubated with B2m-directed guides were stained with anti-mouse 
$\mathrm{H}-2 \mathrm{~Kb}$ (clone AF6-88.5). $\mathrm{H}-2 \mathrm{~Kb}$-negative GFP-positive cells were sorted into single cells on a 96-well plate using FACSAria Fusion (BD Biosciences) and cultured for 2-3 weeks in D10. For cells incubated with Jak1-directed guides, GFP-positive cells were sorted into single cells and cultured for 2-3 weeks in D10. Clones that passed the functional assays

\section{In vitro validation.}

535

DNA was isolated from single-cell WT and knockout clones, and a PCR reaction was done to amplify the edited regions within B2m and Jak1 exons. The PCR products were sequenced by Sanger sequencing, and sequencing results were analyzed with TIDE (Tracking of Indels by Decomposition) analysis ${ }^{33}$ to confirm knockout efficiency. WT and knockout tumor cells were stained for $\mathrm{H} 2-\mathrm{Kb}$ (clone AF6-88.5) to confirm the functional loss of B2m. WT and B2 ${ }^{-/-}$were pulsed with SIINFEKL (30 uM peptide concentration), washed, and coincubated with plate-activated OT1 T cells at 5:1 ratio of effector:target cell. After overnight incubation, cells were washed and stained for CD8 (53-6.7), IFNy (XMG1.2), and GzmB (GB12). For IFNy stimulation assay, WT and knockout tumor cells were incubated with recombinant murine IFNy (Peprotech; $500 \mathrm{EU} / \mathrm{mL}$ ) for 2 days and stained for surface expression of H2-Kb (AF6-88.5) and PD-L1 (10F.9G2).

\section{Tumor RNA isolation and sequencing.}

Mice bearing $\mathrm{WT}, \mathrm{B} 2 \mathrm{~m}^{-/-}$, Jak1 $1^{-/-} \mathrm{MC} 38$ tumors were treated with either aPD1 or IgG1 (100 ug) every 3 or 4 days. After the third administration, approximately $50 \mathrm{mg}$ of tumors were dissected and rapidly frozen with dry ice and IPA. Frozen tumor samples were homogenized in MACS M Tubes (Miltenyi) using the MACS Dissociator (Miltenyi). Total RNA was isolated from the homogenate using the RNeasy Plus Mini Kit (Qiagen). Library preparation with TruSeq RNA Library Prep Kit (Illumina) and mRNA NGS sequencing (40x $10^{6}$ paired end read) were performed by Admera Health (South Plainfield, NJ).

\section{RNA-seq data mapping and visualization.}

Raw FASTQ reads passing quality control (FastQC v0.11.2) were aligned on the mm10 reference genome using STAR aligner (v2.5.2a) with default parameters. Aligned fragments were then counted and annotated using Rsamtools (v3.2) and Cufflinks (v.2.2.1) after a 'dedup' step using BamUtils (v1.0.11). t-SNE embedding results were performed in sklearn (v0.23.1) using all murine genes. Heat maps were plotted with seaborn's (v.0.9.0) clustermap function. Rows were gaussian normalized, and the dendrograms shown for clustering come from hierarchical clustering using Euclidean distance as a metric.

\section{Differential expression and gene set enrichment analysis.}

Differential expression was performed using the edgeR package (v3.24.3) in R using the exactTest method with tagwise dispersion. For mouse data, TMM normalization 
considering mice in all treatment groups was performed to remove library size effect through the calcNormFactors function. For human data ${ }^{11}$, TMM normalization was performed using the two groups being compared. For both datasets, differential expression was performed on Ensembl IDs before mapping to gene names. Then the identified differentially expressed genes were filtered by a list of extracellular and transmembrane endopeptidases queried from UniProt. Gene set enrichment analysis (GSEA) was performed using the fgsea package (v1.8.0) in R. To rank genes, differential expression analysis was first performed on the entire gene set. Genes are then ranked by -sign(logFC)*log(pval). Hallmark gene sets (MSigDB) were used for all GSEA analyses.

Peptide substrate synthesis.

581

582

583

584

585

586

587

588

589

590

591

592

To optimize peptide substrates for target proteases, a library of potential substrates flanked by 5FAM fluorescent dye and DABCYL quencher (5FAM-substrate-

Lys $\{$ DABCYL\}-Amide) was synthesized by Genscript or manufactured in-house using Liberty Blue peptide synthesizer (CEM). The peptide synthesis scale used was $0.025 \mathrm{mM}$, and Low-loading rink amide resin (CEM) was used. Amino acids (Chem-Impex) were resuspended in DMF (0.08 M), as were all synthesis buffers. Activator buffer used was Diisopropylcarbodiimide (DIC; Sigma) (0.25 M) and the activator base buffer was Oxyma (0.25 M; CEM) while the deprotection buffer was Piperidine (20\%; Sigma) supplemented with Oxyma $(0.1 \mathrm{M})$. Crude peptides were purified on 1260 Infinity II HPLC system (Agilent) until a purity of $80 \%$ was achieved. Peptide mass and purity were validated by LC-MS (Agilent) and Autoflex TOF mass spectrometer (Bruker).

\section{Protease substrate library optimization.}

Fluorescently quenched peptide substrates (10 uM) were incubated in manufacturerrecommended buffers at $37^{\circ} \mathrm{C}$ with recombinant proteases (25 nM). Our set of human recombinant proteases included Granzyme A, Granzyme B, MMP1, MMP3, MMP7, MMP9, MMP13, Caspase 1, Caspase 3, Cathepsin G, Cathepsin S (Enzo), human thrombin, human Factor Xla (HaemTech), C1R, Fibroblast Activation Protein alpha/FAP, t-Plasminogen Activator/tPA Protein, and u-Plasminogen Activator/Urokinase (R\&D systems). Sample fluorescence $(E x / E m=488 \mathrm{~nm} / 525 \mathrm{~nm}$ ) were measured for 180 minutes using Cytation 5 plate reader (Biotek). Enzyme cleavage rates were quantified as relative fluorescence increase over time normalized to fluorescence before addition of protease. Hierarchical clustering was performed in python, using log2 fluorescence fold change at 60 minutes. A positive cleavage event was defined as having fluorescence signal more than 2-fold above background. Correlation analysis with Spearman coefficient was done on the cleavage patterns of all peptide substrates for selection of 14 substrates for library construction. These peptide substrates were paired with isobaric mass reporters based on the GluFib peptide (Table 1) and synthesized using Liberty Blue peptide synthesizer (CEM). 
Urinary differentiation of ICB resistant mechanisms.

612 Random forest was used to train classifiers based on urinary reporter signals that 613 differentiate therapeutic response and stratify resistant mechanisms. Response 614 monitoring classifiers were trained on reporter concentration whereas resistance 615 stratifying classifiers were trained on mean normalized reporter concentration. All urine 616 signals were normalized on a per mouse basis by signals on the first dose to performed 617 paired sample analyses. For each classification task, we used five-fold cross validation 618 by randomly left out $1 / 5^{\text {th }}$ samples as the test set and used the remaining samples as 619 training sets. This process was repeated 100 times, and the final performance was 620 generated as the average area under the ROC curve (AUROC) for all train-test results. 621 Comparisons between diagnostic performance was done by two-way paired t-test.

624 Graphs were plotted and appropriate statistical analyses were conducted using 625 GraphPad Prism ( ${ }^{\star} \mathrm{P}<0.05$, ${ }^{\star \star} \mathrm{P}<0.01$, ${ }^{\star \star *} \mathrm{P}<0.001$, ${ }^{\star \star \star \star} \mathrm{P}<0.0001$; central values depict 626 the means, and error bars depict s.e.m.). Measurements were taken from distinct 627 samples. Flow cytometry data were analyzed using FlowJo X (FlowJo, LLC). Power 628 analyses were performed using G*Power 3.1 (HHUD).

Data availability

630 All data supporting the findings of this study are available in the manuscript and its 631 Supplementary Information. Requests for raw data can be addressed to the 632 corresponding author.

633 Code availability

634 All codes used in the manuscript are available upon request to the corresponding author. 


\section{References}

643 1. Ribas, A. \& Wolchok, J. D. Cancer immunotherapy using checkpoint blockade.

$644 \quad$ Science 359, 1350-1355 (2018).

645 2. Sharma, P. \& Allison, J. P. The future of immune checkpoint therapy. Science $\mathbf{3 4 8}$, $646 \quad 56-61(2015)$.

647 3. Sharma, P., Hu-Lieskovan, S., Wargo, J. A. \& Ribas, A. Primary, Adaptive, and Acquired Resistance to Cancer Immunotherapy. Cell 168, 707-723 (2017).

4. Kalbasi, A. \& Ribas, A. Tumour-intrinsic resistance to immune checkpoint blockade. Nat. Rev. Immunol. 1-15 (2019) doi:10.1038/s41577-019-0218-4.

651 5. Nishino, M., Ramaiya, N. H., Hatabu, H. \& Hodi, F. S. Monitoring immune-

652 checkpoint blockade: response evaluation and biomarker development. Nat. Rev. Clin. Oncol. 14, 655-668 (2017).

6. Hodi, F. S. et al. Evaluation of Immune-Related Response Criteria and RECIST v1.1 in Patients With Advanced Melanoma Treated With Pembrolizumab. J. Clin. Oncol. 34, 1510-1517 (2016).

7. Garon, E. B. et al. Pembrolizumab for the Treatment of Non-Small-Cell Lung Cancer. N. Engl. J. Med. 372, 2018-2028 (2015).

8. Nishino, M. et al. Immune-Related Tumor Response Dynamics in Melanoma Patients Treated with Pembrolizumab: Identifying Markers for Clinical Outcome and Treatment Decisions. Clin. Cancer Res. 23, 4671-4679 (2017).

9. Gerwing, M. et al. The beginning of the end for conventional RECIST - novel (2019). 
665

666

667

668

669

670

671

672

673

674

675

676

677

678

679

680

681

682

683

684

685

10. Mandal, R. \& Chan, T. A. Personalized Oncology Meets Immunology: The Path toward Precision Immunotherapy. Cancer Discov. 6, 703-713 (2016).

11. Riaz, N. et al. Tumor and Microenvironment Evolution during Immunotherapy with Nivolumab. Cell 171, 934-949.e16 (2017).

12. Fairfax, B. P. et al. Peripheral CD8 + T cell characteristics associated with durable responses to immune checkpoint blockade in patients with metastatic melanoma. Nat. Med. 26, 193-199 (2020).

13. Valpione, S. et al. Immune awakening revealed by peripheral T cell dynamics after one cycle of immunotherapy. Nat. Cancer 1, 210-221 (2020).

14. Goldberg, S. B. et al. Early Assessment of Lung Cancer Immunotherapy Response via Circulating Tumor DNA. Clin. Cancer Res. 24, 1872-1880 (2018).

15. Kessenbrock, K., Plaks, V. \& Werb, Z. Matrix Metalloproteinases: Regulators of the Tumor Microenvironment. Cell 141, 52-67 (2010).

16. Dudani, J. S., Warren, A. D. \& Bhatia, S. N. Harnessing Protease Activity to Improve Cancer Care. Annu. Rev. Cancer Biol. 2, 353-376 (2018).

17. Martínez-Lostao, L., Anel, A. \& Pardo, J. How Do Cytotoxic Lymphocytes Kill Cancer Cells? Clin. Cancer Res. 21, 5047-5056 (2015).

18. Hilderbrand, S. A. \& Weissleder, R. Near-infrared fluorescence: application to in vivo molecular imaging. Curr. Opin. Chem. Biol. 14, 71-79 (2010).

19. Sanman, L. E. \& Bogyo, M. Activity-Based Profiling of Proteases. Annu. Rev. Biochem. 83, 249-273 (2014). 
686

687

688

689

690

691

692

693

694

695

696

697

698

699

700

701

702

703

704

705

706

707

708

20. Savariar, E. N. et al. Real-time In Vivo Molecular Detection of Primary Tumors and Metastases with Ratiometric Activatable Cell-Penetrating Peptides. Cancer Res. 73, 855-864 (2013).

21. Larimer, B. M. et al. Granzyme B PET Imaging as a Predictive Biomarker of Immunotherapy Response. Cancer Res. 77, 2318-2327 (2017).

22. Kwong, G. A. et al. Mass-encoded synthetic biomarkers for multiplexed urinary monitoring of disease. Nat. Biotechnol. 31, 63-70 (2013).

23. Lin, K. Y., Kwong, G. A., Warren, A. D., Wood, D. K. \& Bhatia, S. N. Nanoparticles That Sense Thrombin Activity As Synthetic Urinary Biomarkers of Thrombosis. ACS Nano 7, 9001-9009 (2013).

24. Warren, A. D., Kwong, G. A., Wood, D. K., Lin, K. Y. \& Bhatia, S. N. Point-of-care diagnostics for noncommunicable diseases using synthetic urinary biomarkers and paper microfluidics. Proc. Natl. Acad. Sci. 111, 3671-3676 (2014).

25. Kwong, G. A. et al. Mathematical framework for activity-based cancer biomarkers. Proc. Natl. Acad. Sci. 112, 12627-12632 (2015).

26. Mac, Q. D. et al. Non-invasive early detection of acute transplant rejection via nanosensors of granzyme B activity. Nat. Biomed. Eng. 3, 281-291 (2019).

27. Kirkpatrick, J. D. et al. Urinary detection of lung cancer in mice via noninvasive pulmonary protease profiling. Sci. Transl. Med. 12, (2020).

28. Efremova, M. et al. Targeting immune checkpoints potentiates immunoediting and changes the dynamics of tumor evolution. Nat. Commun. 9, 32 (2018).

29. Desnoyers, L. R. et al. Tumor-Specific Activation of an EGFR-Targeting Probody Enhances Therapeutic Index. Sci. Transl. Med. 5, 207ra144-207ra144 (2013). 
30. Strohl, W. R. Fusion Proteins for Half-Life Extension of Biologics as a Strategy to Make Biobetters. BioDrugs 29, 215-239 (2015).

31. Duraiswamy, J., Kaluza, K. M., Freeman, G. J. \& Coukos, G. Dual Blockade of PD-1 and CTLA-4 Combined with Tumor Vaccine Effectively Restores T-Cell Rejection Function in Tumors. Cancer Res. 73, 3591-3603 (2013).

32. Selby, M. J. et al. Preclinical Development of Ipilimumab and Nivolumab Combination Immunotherapy: Mouse Tumor Models, In Vitro Functional Studies, and Cynomolgus Macaque Toxicology. PLOS ONE 11, e0161779 (2016).

34. Liberzon, A. et al. The Molecular Signatures Database Hallmark Gene Set

33. Brinkman, E. K., Chen, T., Amendola, M. \& van Steensel, B. Easy quantitative

35. Schwartz, L. H. et al. RECIST 1.1 - Update and Clarification: From the RECIST Committee. Eur. J. Cancer Oxf. Engl. 1990 62, 132-137 (2016).

36. Arlot, S. \& Celisse, A. A survey of cross-validation procedures for model selection. Stat. Surv. 4, 40-79 (2010).

37. Patel, S. P. \& Kurzrock, R. PD-L1 Expression as a Predictive Biomarker in Cancer Immunotherapy. Mol. Cancer Ther. 14, 847-856 (2015).

38. Chan, T. A. et al. Development of tumor mutation burden as an immunotherapy biomarker: utility for the oncology clinic. Ann. Oncol. 30, 44-56 (2019). based immunotherapy. Science 362, (2018). 
732 40. Chang, L., Chang, M., Chang, H. M. \& Chang, F. Microsatellite Instability: A

733 Predictive Biomarker for Cancer Immunotherapy. Appl. Immunohistochem. Mol.

$734 \quad$ Morphol. 26, e15 (2018).

735 41. Bratman, S. V. et al. Personalized circulating tumor DNA analysis as a predictive

736 biomarker in solid tumor patients treated with pembrolizumab. Nat. Cancer 1, 873-

$737 \quad 881$ (2020).

738 42. Tietze, J. K. et al. The proportion of circulating CD45RO+CD8+ memory T cells is

739 correlated with clinical response in melanoma patients treated with ipilimumab. Eur.

$740 \quad$ J. Cancer 75, 268-279 (2017).

741 43. Chen, P.-L. et al. Analysis of Immune Signatures in Longitudinal Tumor Samples

$742 \quad$ Yields Insight into Biomarkers of Response and Mechanisms of Resistance to

743 Immune Checkpoint Blockade. Cancer Discov. 6, 827-837 (2016).

744 44. Tumeh, P. C. et al. PD-1 blockade induces responses by inhibiting adaptive immune

745 resistance. Nature 515, 568-571 (2014).

746 45. Jiang, P. et al. Signatures of T cell dysfunction and exclusion predict cancer

747 immunotherapy response. Nat. Med. 24, 1550-1558 (2018).

748 46. Larimer, B. M. et al. The Effectiveness of Checkpoint Inhibitor Combinations and

749 Administration Timing Can Be Measured by Granzyme B PET Imaging. Clin. Cancer

$750 \quad$ Res. 25, 1196-1205 (2019).

751 47. Zhang, X. et al. Hepatitis B virus reactivation in cancer patients with positive

752 Hepatitis B surface antigen undergoing PD-1 inhibition. J. Immunother. Cancer 7,

$35322(2019)$ 
48. Del Castillo, M. et al. The Spectrum of Serious Infections Among Patients Receiving

755

756

757

758

759

760

761

762

763

764

765

766

767

768

769

770

771

772

773

774

775

776

Immune Checkpoint Blockade for the Treatment of Melanoma. Clin. Infect. Dis. 63, 1490-1493 (2016).

49. Fujita, K. et al. Emerging concerns of infectious diseases in lung cancer patients receiving immune checkpoint inhibitor therapy. Respir. Med. 146, 66-70 (2019).

50. Aguilera, T. A., Olson, E. S., Timmers, M. M., Jiang, T. \& Tsien, R. Y. Systemic in vivo distribution of activatable cell penetrating peptides is superior to that of cell penetrating peptides. Integr. Biol. 1, 371-381 (2009).

51. Whitley, M. J. et al. A mouse-human phase 1 co-clinical trial of a protease-activated fluorescent probe for imaging cancer. Sci. Transl. Med. 8, 320ra4-320ra4 (2016).

52. Timmer, J. C. \& Salvesen, G. S. Caspase substrates. Cell Death Differ. 14, 66-72 (2007).

53. Poreba, M. et al. Unnatural amino acids increase sensitivity and provide for the design of highly selective caspase substrates. Cell Death Differ. 21, 1482-1492 (2014).

54. Rut, W. et al. Recent advances and concepts in substrate specificity determination of proteases using tailored libraries of fluorogenic substrates with unnatural amino acids. Biol. Chem. 396, 329-337 (2015).

55. Miller, M. A. et al. Proteolytic Activity Matrix Analysis (PrAMA) for simultaneous determination of multiple protease activities. Integr. Biol. 3, 422-438 (2011).

56. Zhuang, Q., Holt, B. A., Kwong, G. A. \& Qiu, P. Deconvolving multiplexed protease signatures with substrate reduction and activity clustering. PLOS Comput. Biol. 15, e1006909 (2019) 
57. Austin, R. J. et al. TriTACs, a Novel Class of T-Cell-Engaging Protein Constructs

778 Designed for the Treatment of Solid Tumors. Mol. Cancer Ther. 20, 109-120 (2021).

779 58. Triplett, T. A. et al. Reversal of indoleamine 2,3-dioxygenase-mediated cancer

780 immune suppression by systemic kynurenine depletion with a therapeutic enzyme. Nat. Biotechnol. 36, 758-764 (2018). Enzymology vol. 118 742-766 (Academic Press, 1986). 
Acknowledgments: This work was funded by the NIH Director's New Innovator Award DP2HD091793 and the National Cancer Institute R01 grant 5R01CA237210. Q.D.M. and A.S. are supported by the NSF Graduate Research Fellowships Program (Grant No. 800 DGE-1650044). G.A.K. holds a Career Award at the Scientific Interface from the 801 Burroughs Wellcome Fund. P.Q. is an ISAC Marylou Ingram Scholar and a Carol Ann 802 and David D. Flanagan Faculty Fellow. This work was performed in part at the Georgia 803 Tech Institute for Electronics and Nanotechnology, a member of the National 804 Nanotechnology Coordinated Infrastructure, which is supported by the National Science 805 Foundation (Grant ECCS-1542174). This content is solely the responsibility of the authors 806 and does not necessarily represent the official views of the National Institutes of Health.

807 The authors would like to thank the staffs at Georgia Tech mass spectrometry core, flow 808 cytometry analysis core, and the animal facility for their assistance in performing our 809 studies.

810 Author contributions: Q.D.M., J.R.B, and G.A.K. conceived of the idea. Q.D.M., C.X., 811 J.R.B, A.S., H.P., F-Y.S., S.Z.S., P.Q., and G.A.K. designed experiments and interpreted 812 results. Q.D.M., C.X., J.R.B, A.S., H.P., F-Y.S., S.Z.S., H.S., A.M.H., and T.T.L. carried 813 out the experiments. Q.D.M., A.S., and G.A.K. wrote the manuscript.

814 Correspondence and requests for materials should be addressed to the corresponding 815 author (G.A.K).

816 Ethical compliance. All authors have complied with relevant ethical regulations while 817 conducting this study. 
818 Competing interests: G.A.K. is co-founder of and serves as consultant to Glympse Bio,

819 which is developing products related to the research described in this paper. This study

820 could affect his personal financial status. The terms of this arrangement have been

821 reviewed and approved by Georgia Tech in accordance with its conflict-of-interest

822 policies. Q.D.M., J.R.B., and G.A.K are listed as inventors on a patent application

823 pertaining to the results of the paper. The patent applicant is the Georgia Tech Research

824 Corporation. The names of the inventors are Quoc Mac, James Bowen, and Gabriel

825 Kwong. The application number is PCT/US2019/050530. The patent is currently

826 pending/published (publication number WO2020055952A1). The mass-barcoded

827 antibody-sensor conjugates and related applications are covered in this patent.

828

829

830

831

832

833

834

835

836

837

838

839 


\section{$841 \quad$ Figures}
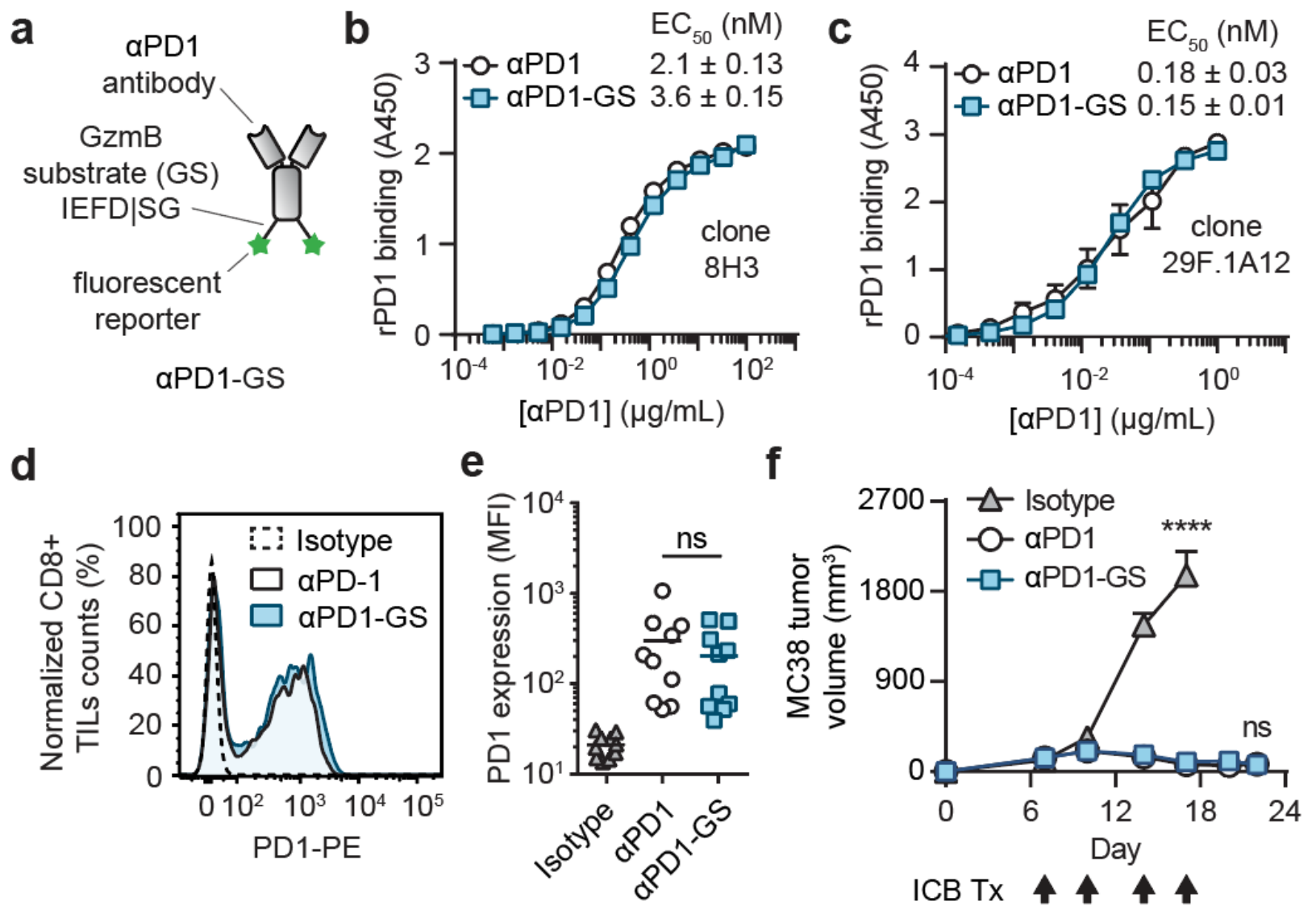

843 Figure 1 | Antibody binding and therapeutic efficacy are unaffected by peptide

844 conjugation. a, aPD1-GzmB sensor conjugates (aPD1-GS) consist of aPD1 therapeutic

845 antibody decorated with reporter-labeled GzmB peptide substrates (GS; AA sequence:

846 IEFDSG). b, ELISA assays comparing binding affinity of aPD1-GS with unconjugated

847 aPD1 using the mouse aPD1 clone 8H3 (log(agonist) vs. normalized response fitting

848 function, $n=3)$. c, ELISA assays comparing binding affinity of aPD1-GS with

849 unconjugated aPD1 using the rat aPD1 clone 29F.1A12 (log(agonist) vs. normalized

850 response fitting function, $n=3$ ). $\mathbf{d}$, Representative flow cytometry histogram showing PD-

8511 expression of CD8+ TILs isolated from MC38 tumors. The same sample was divided

852 and stained with either aPD1-GS, aPD1, or IgG1 isotype control. e, Quantified plot of PD- 
8531 expression showing the median fluorescence intensity (MFI) of samples stained with

854 either aPD1-GS, aPD1, or IgG1 isotype control (one-way ANOVA with Tukey's post-test

855 and correction for multiple comparisons, $n s=$ not significant, $n=10) . \mathbf{f}$, Tumor growth

856 curves of MC38 tumors treated with aPD1-GS, aPD1, or IgG1 isotype control (two-way

857 ANOVA with Tukey's post-test and correction for multiple comparisons, ${ }^{* \star * * P}<0.0001, n$

$858=6)$.

859

860

861

862

863

864

865

866

867

868

869

870

871

872

873

874

875 
a

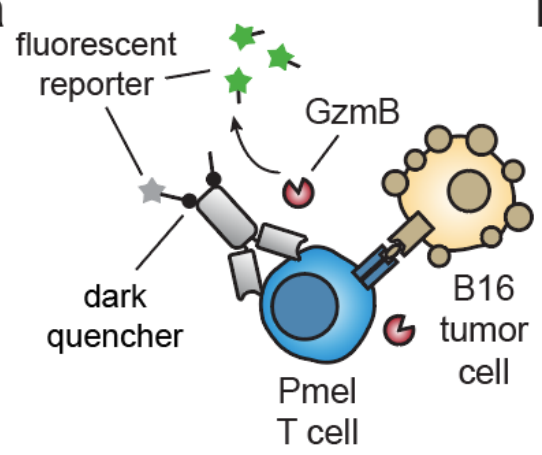

b

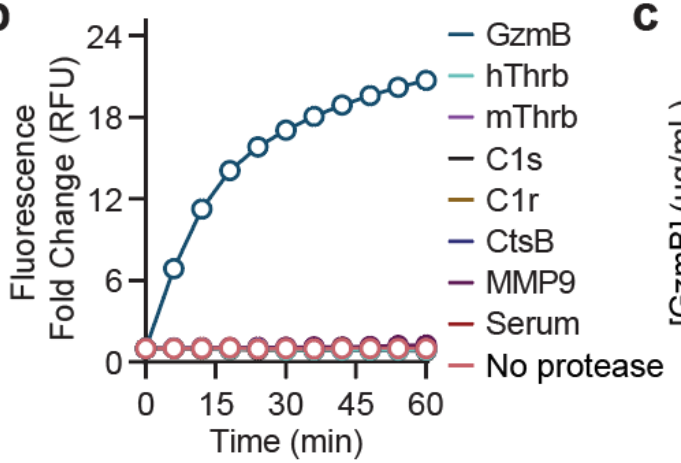

C

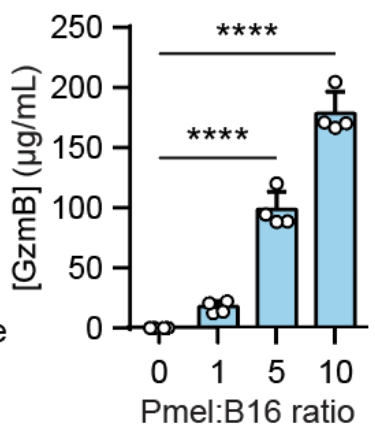

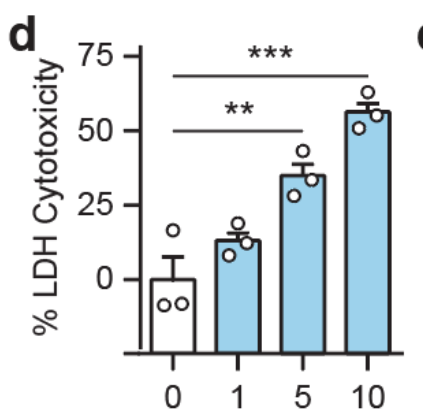

Pmel:B16 ratio

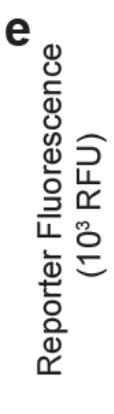

876

877

878

879

880

881

882

883

884

885

886

887

888

889

Figure 2 | Sensing $T$ cell killing of tumor cells by antibody-GzmB sensor

conjugates. a, aPD1 antibody was conjugated with fluorescently-quenched peptide

substrates for GzmB. Upon incubating these conjugates with transgenic Pmel T cells and

B16 tumor cells, secreted GzmB cleaved peptide substrates, separating the fluorescent

reporter from the internal quencher and resulting in an increase in sample fluorescence.

b, In vitro protease cleavage assays showing normalized fluorescence of aPD1-GS after

incubation with recombinant GzmB (blue), mouse serum (red), and other bystander

proteases $(n=3)$. c, ELISA quantification of GzmB from $T$ cell killing assays in which

Pmel T cells were incubated with B16 target cells at different T cell to target cell ratios

(one-way ANOVA with Dunnett's post-test and correction for multiple comparisons, ${ }^{\star \star \star \star P}$

$<0.0001, \mathrm{n}=4$ ). $\mathbf{d}$, Bar plot quantifying percent of cell cytotoxicity as measured by LDH

assay from cocultures of Pmel T cells with B16 target cells (one-way ANOVA with

Dunnett's post-test and correction for multiple comparisons, ${ }^{\star \star \star} P<0.001, n=3$ ). e, 
890 Activity assays showing sample fluorescence after incubating aPD1-GS, aPD1, and an 891 aPD1 conjugate with control substrates (aPD1-CtrlSub) with cocultures of Pmel T cells 892 with B16 target cells (two-way ANOVA with Tukey's post test and correction for multiple 893 comparisons, $\left.{ }^{\star \star \star *} \mathrm{P}<0.0001, \mathrm{n}=3\right)$. f, Activity assays showing sample fluorescence after 894 incubating aPD1-GS with cocultures of Pmel or OT1 transgenic T cells with B16 target 895 cells (two-way ANOVA with Tukey's post test and correction for multiple comparisons, $896 * \star \star \star P<0.0001, \mathrm{n}=3)$. 

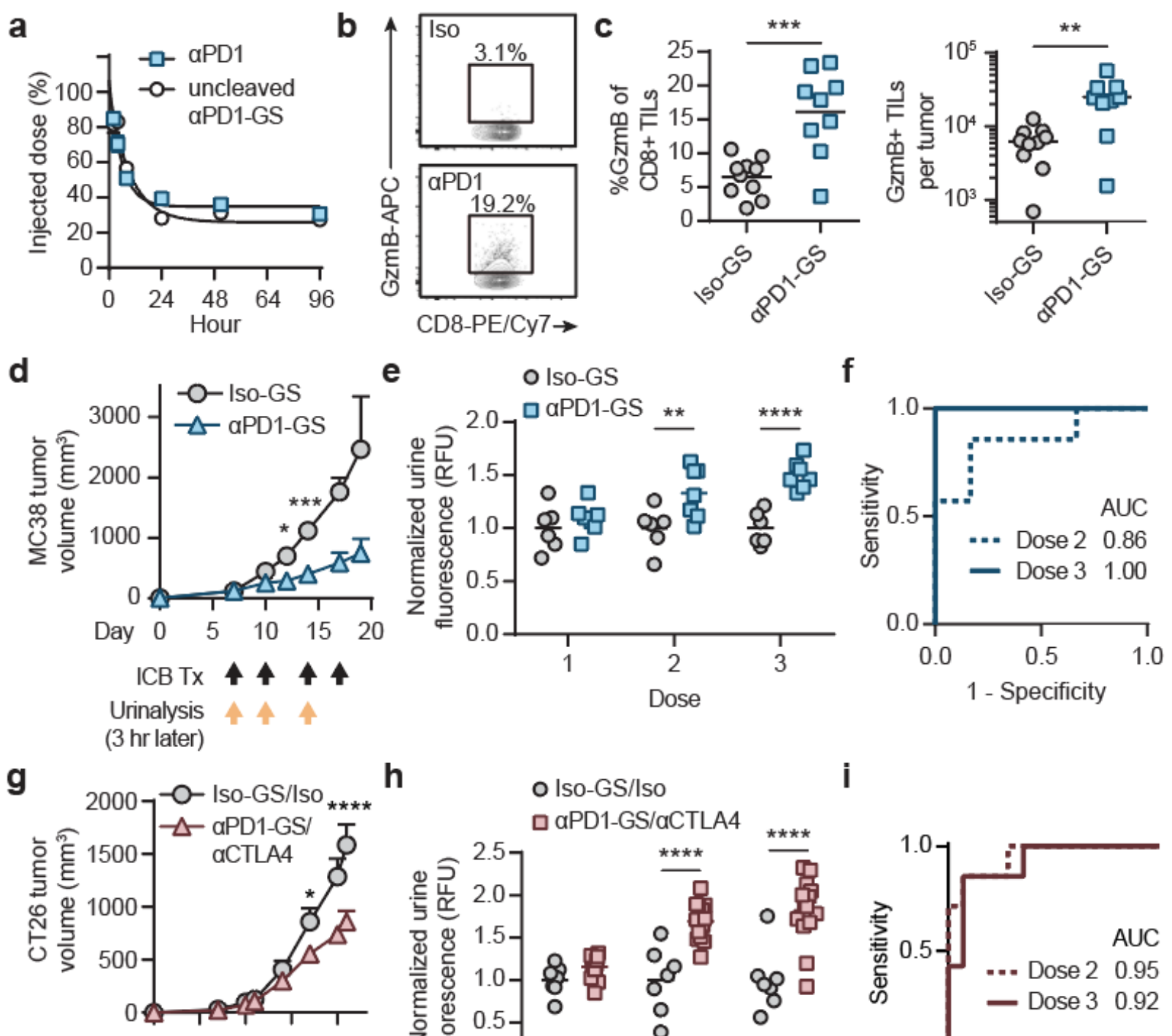

Day $0 \quad 5 \quad 10 \quad 15 \quad 20$

ICB Tx

Urinalysis

912

h
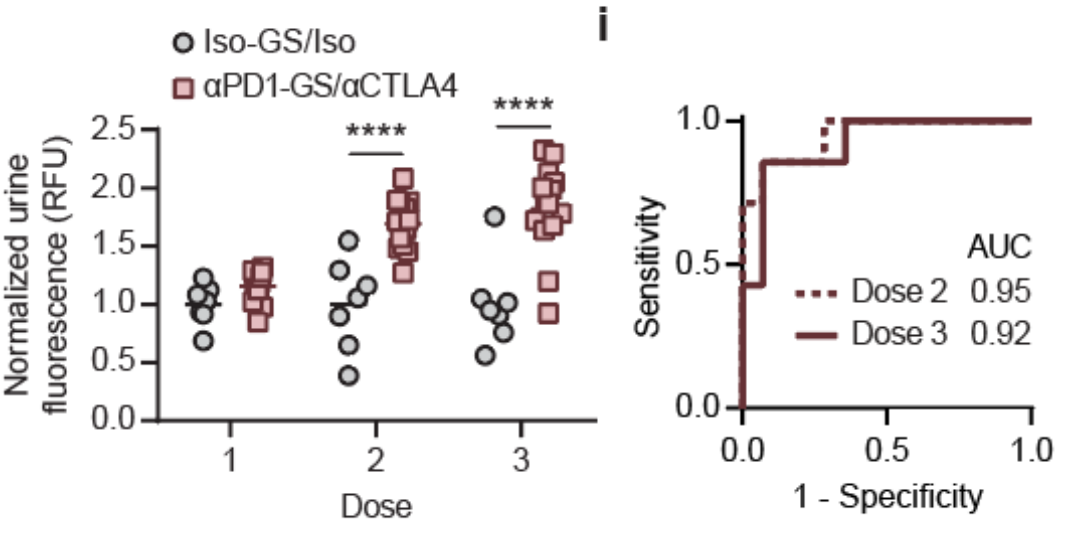

913 Fig 3 | Urinary detection of ICB therapeutic response by administration of antibody-

914 GzmB sensor conjugates. a, Half-life measurements of intact aPD1-GS and

915 unconjugated aPD1 antibody (one phase decay fitting function, $n=3$ ). b, Representative

916 flow cytometry plots showing intracellular GzmB expression of CD8+ TILs from MC38

917 tumors treated with either aPD1-GS or IgG1 isotype antibody conjugated with the GzmB

918 peptide substrates (Iso-GS). c, Quantified plots showing percentages of GzmB+ cells 
919 within the CD8+ TILs or the numbers of GzmB+CD8+ TILs that were isolated from MC38

920 tumors treated with either aPD1-GS or Iso-GS (two-sided Student's t-test, $\mathrm{n}=9-10$ ). d,

921 Tumor growth curves of MC38 tumor bearing mice treated with either aPD1-GS or Iso-

922 GS (two-way ANOVA with Sidak's post test and correction for multiple comparisons, ${ }^{* \star} \mathrm{P}$

$923<0.001, n=6-7)$. Black arrows denote the treatment time points. e, Left: normalized urine

924 fluorescence of mice with MC38 tumors after each administration of aPD1-GS or Iso-GS

925 (two-way ANOVA with Sidak's post test and correction for multiple comparisons, ${ }^{\star \star \star \star} \mathrm{P}<$

926 0.0001, $\mathrm{n}=6-7$ ). $\mathbf{f}$, Receiver-operating-characteristic (ROC) analysis showing the

927 diagnostic specificity and sensitivity in differentiating between mice treated with aPD1-GS

928 vs. Iso-GS using urine signals on the second $(A \cup C=0.857,95 \% \mathrm{Cl}=0.643-1.00)$ or the

929 third dose $(A \cup C=1.00,95 \% \mathrm{Cl}=1.00-1.00) . \mathbf{g}$, Tumor growth curves of CT26 tumor

930 bearing mice treated with combination therapy of aPD1-GS and aCTLA4 or combination

931 of matched isotype controls (two-way ANOVA with Sidak's post test and correction for

932 multiple comparisons, $\left.{ }^{\star \star \star \star} \mathrm{P}<0.0001, \mathrm{n}=7-14\right)$. Black arrows denote the treatment time

933 points. h, Normalized urine fluorescence of mice with CT26 tumors after each

934 administration of aPD1-GS and aCTLA4 or matched isotype controls (two-way ANOVA

935 with Sidak's post test and correction for multiple comparisons, $\left.{ }^{\star \star \star \star} \mathrm{P}<0.0001, \mathrm{n}=7-14\right)$.

936 i, ROC analysis showing the diagnostic specificity and sensitivity of aPD1-GS in

937 differentiating between responders to ICB combination therapy from off-treatment

938 controls using urine signals on the second ( $\mathrm{AUC}=0.949,95 \% \mathrm{Cl}=0.856-1.00$ ) or the

939 third dose $(\mathrm{AUC}=0.92,95 \% \mathrm{Cl}=0.795-1.00)$ 
a

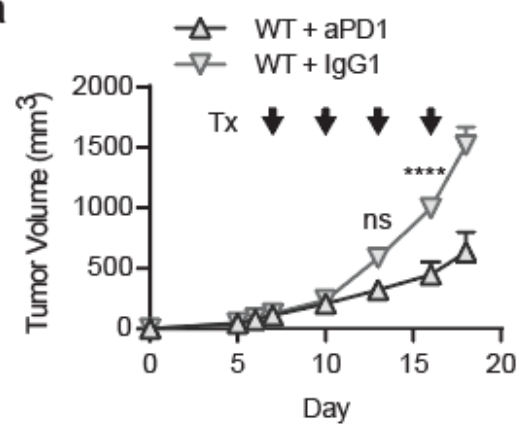

b

- WT + aPD1 $\quad W T+\lg$ G1

- $\mathrm{B} 2 \mathrm{~m}^{-1-}+\mathrm{aPD} 1 \bullet \mathrm{B}^{2} \mathrm{~m}^{-1-}+\mathrm{lgG} 1$

- Jak1 $1^{-/}+\mathrm{aPD} 1$ Jak1 $1^{-/}+\lg \mathrm{G} 1$

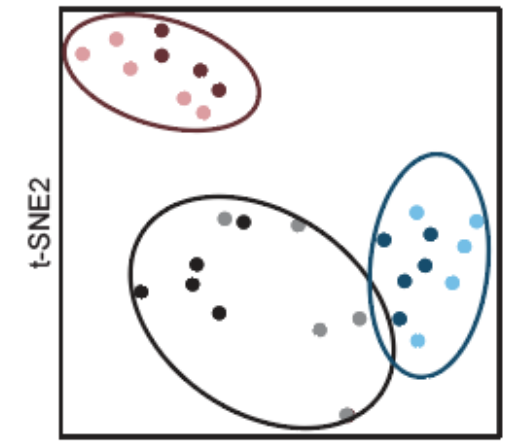

t-SNE1

d
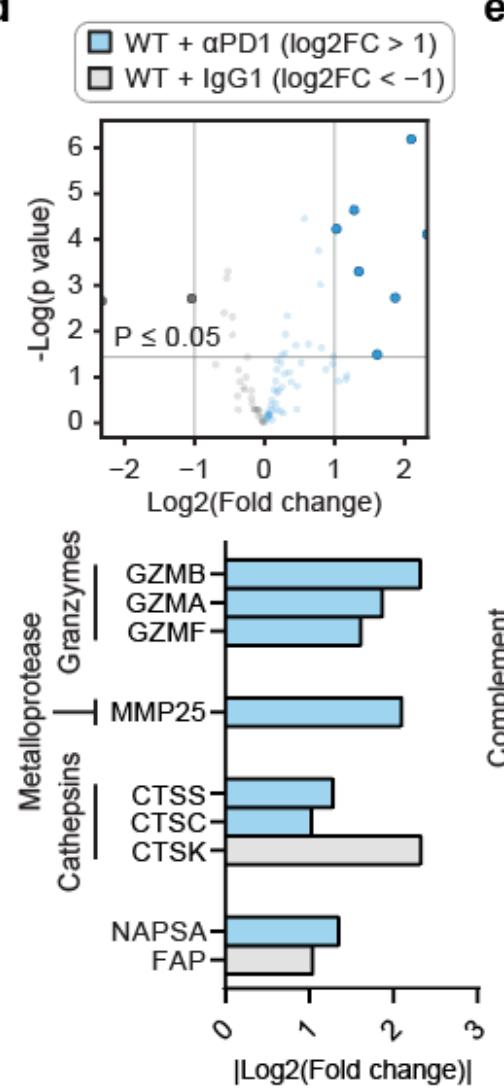

940

e
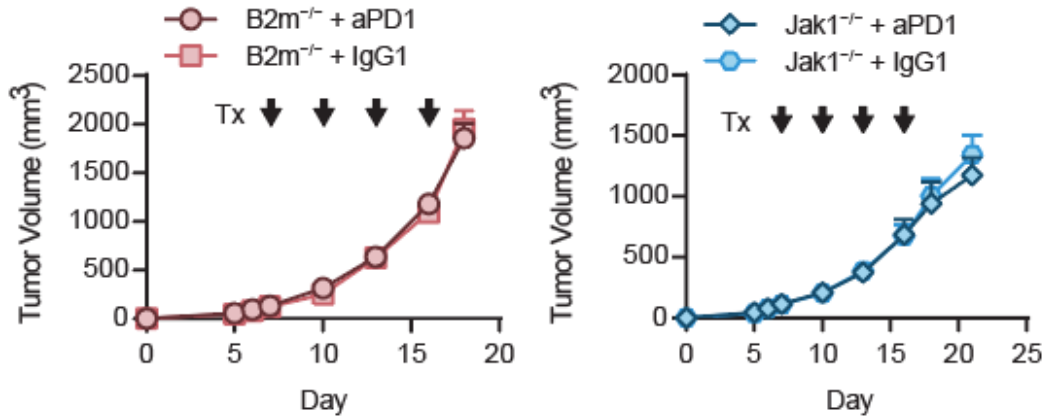

c
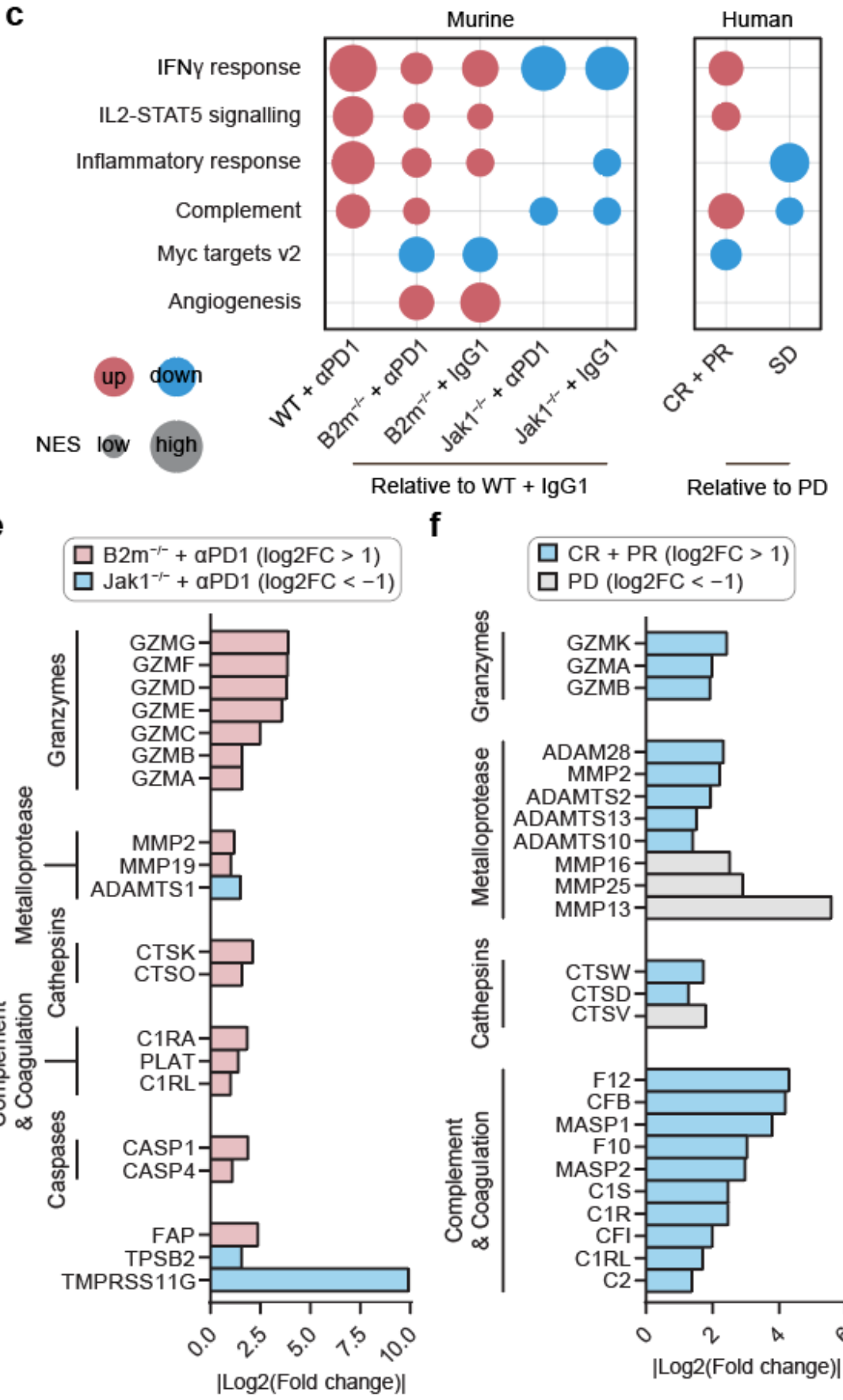

$\mathbf{f}$
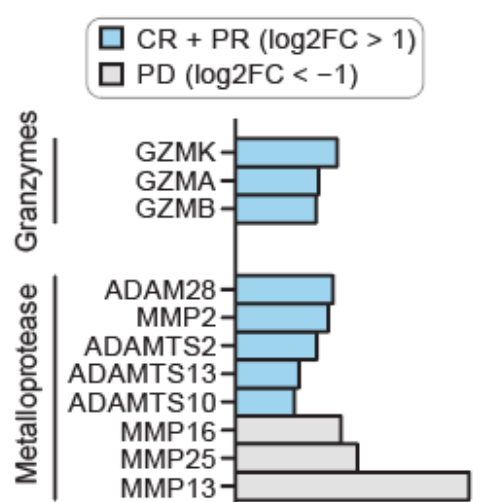

PD $(\log 2 \mathrm{FC}<-1)$

嗀|

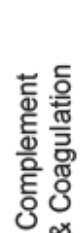

CTSW-

CTSV-

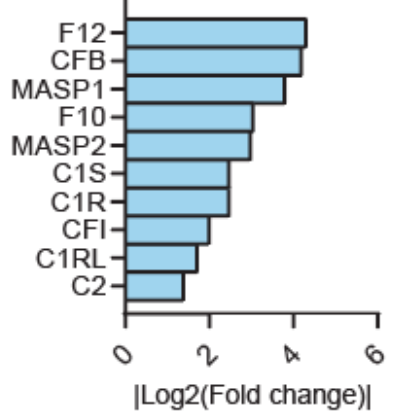


941 Figure 4 | Proteases are dysregulated in ICB response and resistance. a, Tumor

942 growth curves of mice bearing WT (left), B2m-1- (middle), or Jak1 ${ }^{-/-}$(right) MC38 tumor

943 treated with aPD1 or matched IgG1 control (two-way ANOVA with Sidak's post test and

944 correction for multiple comparisons, $\left.{ }^{\star \star \star *} \mathrm{P}<0.0001, \mathrm{n}=15-25\right)$. Black arrows denote the

945 treatment time points. b, t-SNE plot showing global transcriptional profiles of WT, B2m ${ }^{-/-}$,

946 and Jak1 ${ }^{-/-}$MC38 tumors treated with aPD1 or lgG1 isotype control $(n=5)$. c, Left: GSEA

947 comparing gene set signatures of all mouse tumors and treatment groups relative to WT

948 tumors receiving isotype control treatment $(n=5) .6$ gene sets were shown from the

949 canonical Hallmark gene sets ${ }^{34}$, with 4 immune- and 2 tumor-associated gene sets. Only

950 the gene sets that are significantly different (false discovery rate $<0.05$ ) between the two

951 groups being compared were shown. Red color indicates upregulation in the first group,

952 and blue indicates downregulation. The size of the circle represents the nominal

953 enrichment score (NES). Right: similar GSEA analyses using human data from melanoma

954 patients treated with aPD1 monotherapy ${ }^{11}$. Gene set signatures of the two patient groups

955 (Complete Response (CR) + Partial Response (PR), and Stable Disease (SD)) were

956 compared to patients with Progressive Disease (PD). d, Top: Volcano plots summarizing

957 the extracellular and transmembrane proteases differentially expressed between WT

958 MC38 tumors treated with aPD1 or $\lg 1(n=5)$. The threshold for differentially expressed

959 genes (opaque dots) was defined as $P$ value $\leq 0.05$ and $\| \log 2$ (fold change) $\mid \geq 1$. Bottom:

960 waterfall plot showing the fold changes in transcript levels of proteases that are

961 differentially expressed between these two groups. The proteases are grouped into the

962 families of interest while the remaining are greyed out. e, Waterfall plot showing the fold

963 changes in transcript levels of proteases that are differentially expressed between aPD1 
964 treated B2m ${ }^{-/-}$and Jak1 $1^{-1-}$ tumors $(n=5)$. $\mathbf{f}$, Waterfall plot showing the fold changes in

965 transcript levels of proteases that are differentially expressed between human tumors

966 from responders (CR + PR) and non-responders (PD).

967

968

969 
a

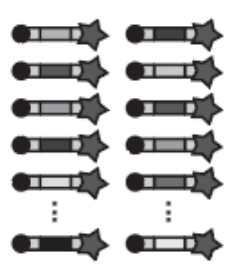

66 fluorogenic substrates

b

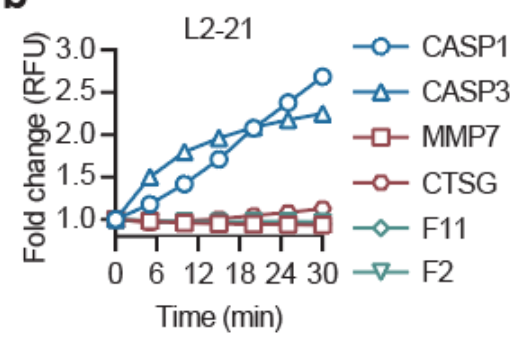

c
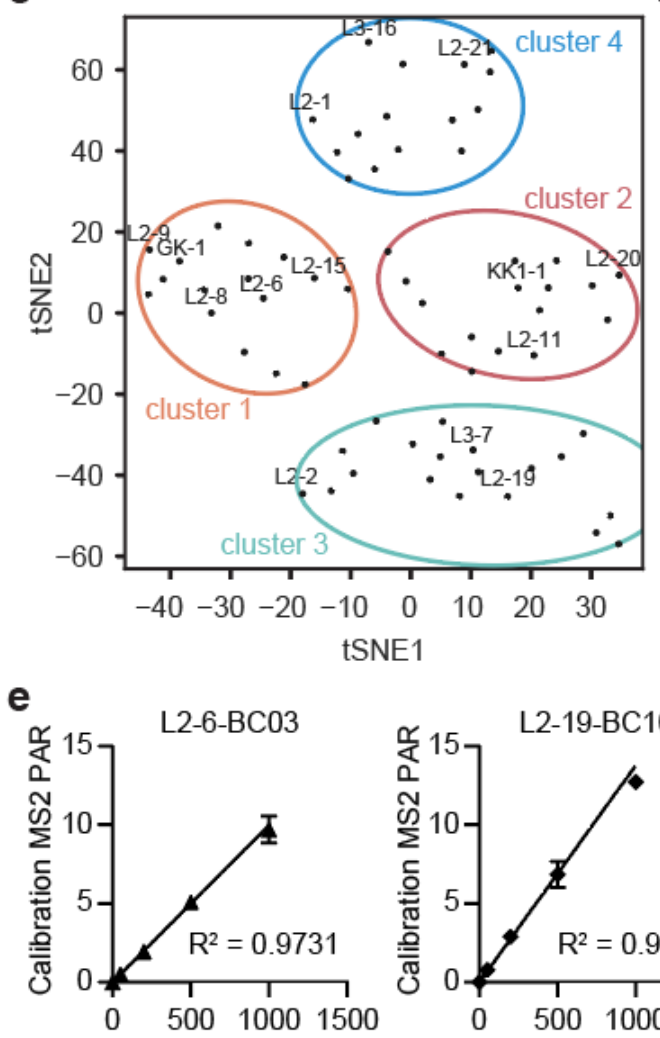

[Substrate] (ng/mL)

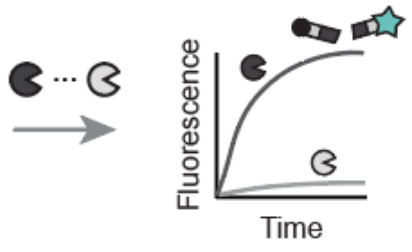

17 target proteases quantify cleavage fluorescence

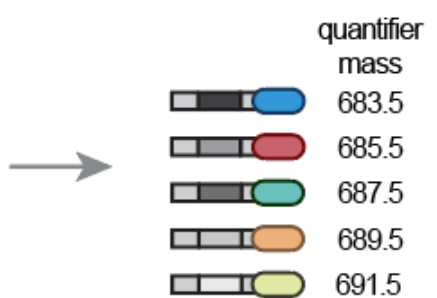

14 mass-barcoded substrates

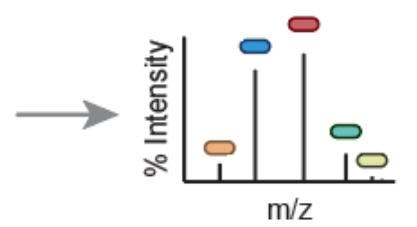

LC/MS-MS analysis
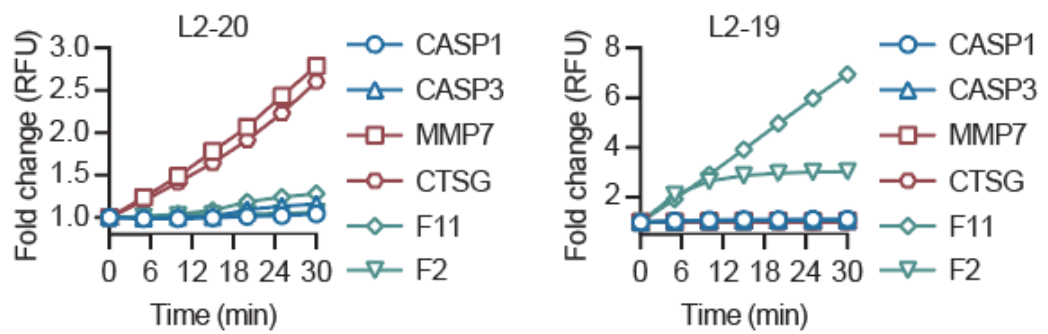

d

970

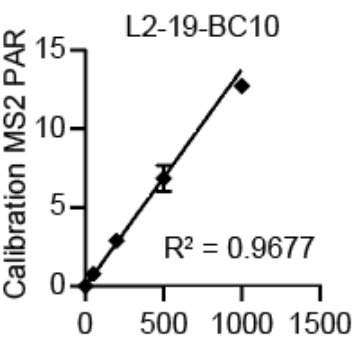

[Substrate] (ng/mL)
L2-1

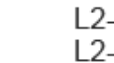

L2-9

GK-1

\% L2-8

L2-15

KK1-1

L2-20

L2-11

L2-19

L3-16

L3-7

L2-21
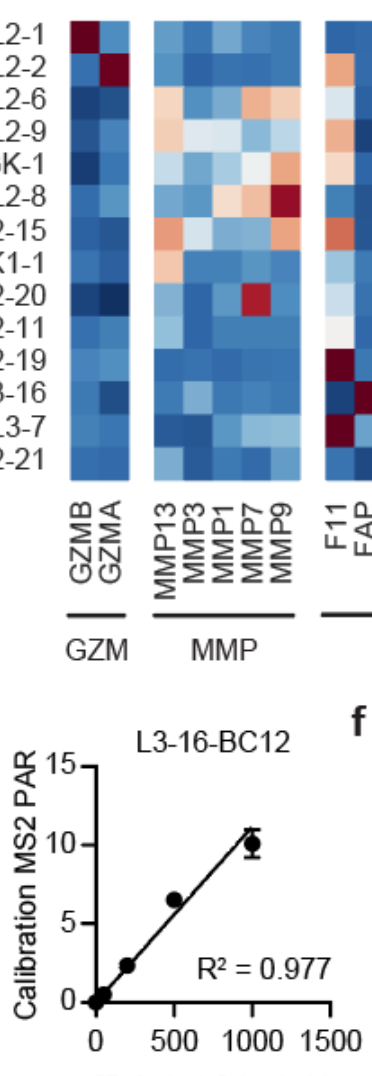

[Substrate] (ng/mL)
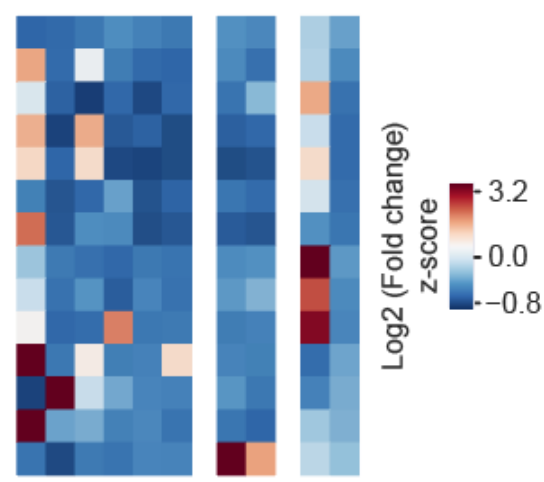

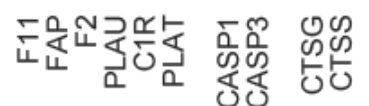

CASP CTS
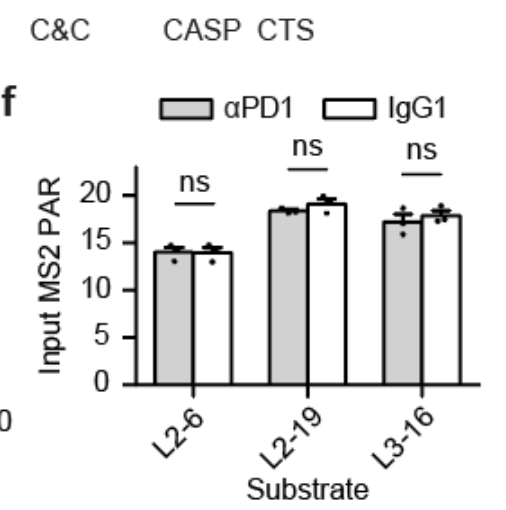

971 Figure 5 | Mass-barcoded peptide sensors for multiplexed detection of protease

972 activity. a, Schematic of the peptide substrate screen to identify candidate substrates for 
974 recombinant proteases of interest. Each cleavage trace represents the average of 3

975 independent replicates. c, t-SNE plot showing unsupervised clustering of 66 candidate

976 substrates into major clusters. d, Heat map summarizing the log2 fold change in

977 fluorescence of 14 selected substrates at $60 \mathrm{~min}$ after addition of the respective

978 recombinant protease $(n=3)$. Signals were row-normalized before plotting. e, Calibration

979 curves of mass barcodes as quantified by LC-MS/MS. MS2 peak area from each mass

980 barcode used to label representative substrates is normalized by peak area of an internal

981 standard to obtain peak-area-ratio (PAR). f, Bar plot showing corresponding mass

982 reporter signals (PAR) from mixtures of aPD1- or IgG1-peptide conjugates (two-way

983 ANOVA with Tukey's post test and correction for multiple comparisons, $n=3$ ). 
a

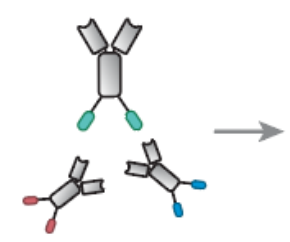

Library of 14-plex mass-encoded mAb-sensor conjugates
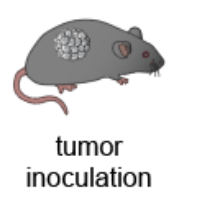

Day

$\downarrow$ aPD1/lgG1-sensor treatment
$\downarrow$ Urine collection (3 hours)
WT MC38

$\mathrm{B} 2 \mathrm{~m}^{-/-} \mathrm{MC} 38$

Jak1 ${ }^{-1-}$ MC38

$\downarrow \bullet \bullet \longrightarrow$

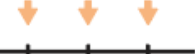

13

)

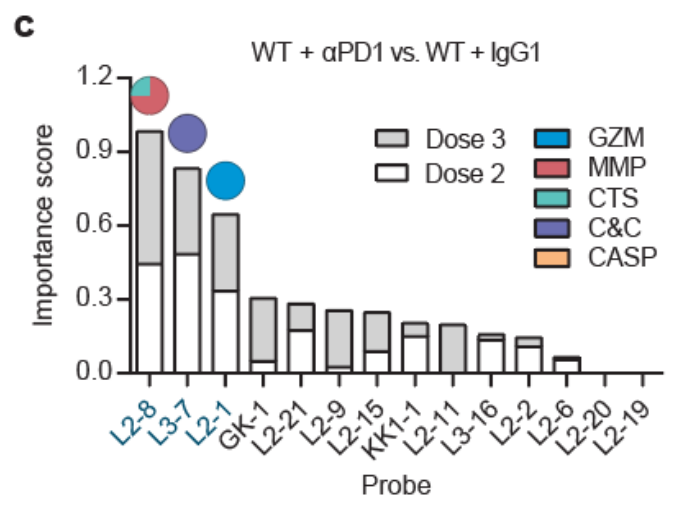

e
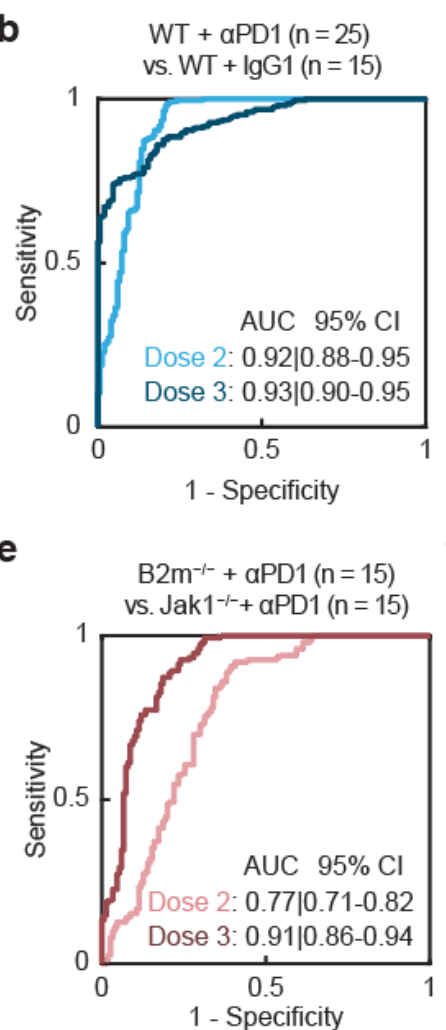

$$
\text { f }
$$

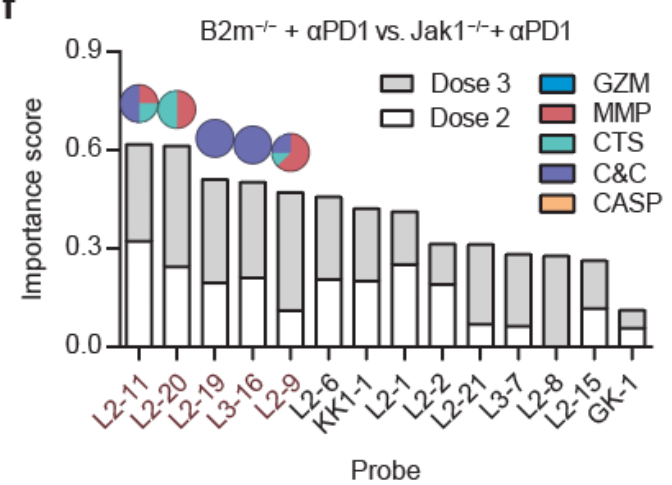

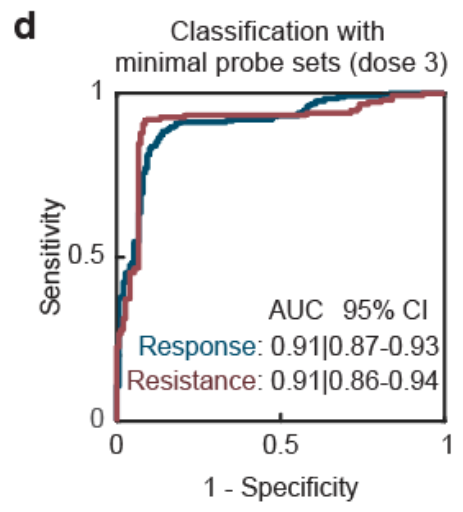

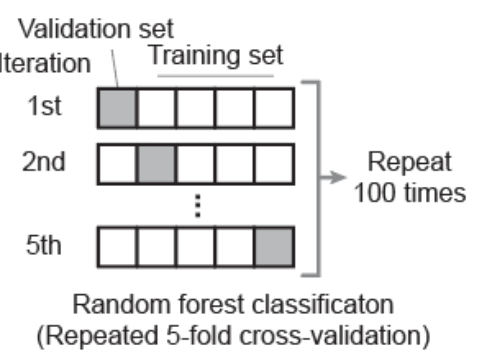

g

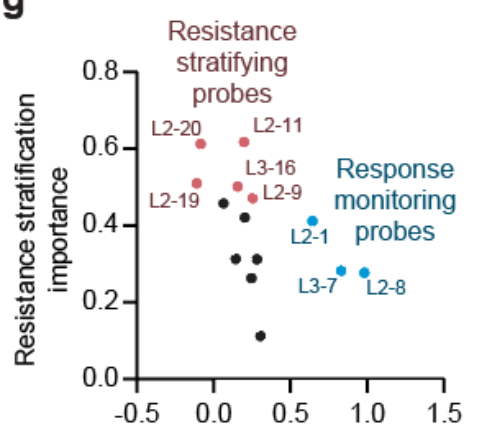

Response monitoring importance

Figure 6 | Urinary classification of ICB response and resistance. a, Schematic of our

987 pipeline to develop urinary classifiers of ICB response and resistance. $\mathbf{b}$, Area under the

988 ROC curve (AUC) analysis showing the diagnostic specificity and sensitivity of random

989 forest classifiers based on INSIGHT library in differentiating between aPD1-treated WT

990 tumors $(n=25)$ and IgG1-treated controls $(n=15)$ using urine signals on dose 2 (AUC =

$9910.92,95 \% \mathrm{Cl}=0.88-0.95)$ or dose $3(\mathrm{AUC}=0.93,95 \% \mathrm{Cl}=0.90-0.95)$. c , Feature

992 importance analysis revealing the probes that are important for response monitoring.

993 Probes with higher important scores, produced by random forest, contribute more to the 
994 diagnostic performance. The pie charts above individual probes show the protease 995 families that are monitored by each probe. $\mathbf{d}$, AUC analysis of random forest classifiers 996 based on the top 3 probes (L2-8, L3-7, L2-1) for response monitoring (AUC $=0.91,95 \%$ $997 \mathrm{Cl}=0.87-0.93)$ and the top 5 probes $(\mathrm{L} 2-11, \mathrm{~L} 2-20, \mathrm{~L} 2-19, \mathrm{~L} 3-16$, and L2-9) for resistance 998 stratification $(\mathrm{AUC}=0.91,95 \% \mathrm{Cl}=0.86-0.94)$. e, $\mathrm{AUC}$ analysis of random forest 999 classifiers based on INSIGHT library in differentiating between aPD1-treated B2m ${ }^{-/-}(n=$ 1000 15) from Jak1 ${ }^{-/-}$MC38 $(n=15)$ tumors using urine signals on dose 2 (AUC $=0.77,95 \%$ $1001 \mathrm{Cl}=0.71-0.82)$ or dose $3(\mathrm{AUC}=0.91,95 \% \mathrm{Cl}=0.86-0.94) . \mathrm{f}$, Feature importance 1002 analysis revealing the probes that are important for resistance stratification. $\mathbf{g}$, Scatter 1003 plot showing feature important scores of all 14 probes in the INSIGHT panel for response 1004 monitoring and resistance stratification. The highlighted probes belong to the minimal 1005 probe sets that achieve comparable diagnostic performance in these classification tasks 1006 as compared to using the entire INSIGHT panel.

1007

1008

1009

1010

1011

1012

1013

1014

1015 


\begin{tabular}{|c|c|c|c|c|}
\hline Name & Substrate & Barcode & $\begin{array}{l}\text { Precursor } \\
\text { Mass (MS1) }\end{array}$ & $\begin{array}{c}\text { Quantifier } \\
\text { Mass (MS2) }\end{array}$ \\
\hline L2-1 & IEFDSG & BC01 & 806.5 & 683.5 \\
\hline L2-2 & VANRSAS & BC02 & 808.5 & 683.5 \\
\hline L2-6 & RPLALWRSD & $\mathrm{BC03}$ & 808.5 & 685.5 \\
\hline L2-8 & RPLGLAGK & $\mathrm{BCO4}$ & 808.5 & 687.5 \\
\hline L2-9 & PLAQAVRS & BC05 & 810.5 & 683.5 \\
\hline L2-11 & AFRFSQK & BC06 & 810.5 & 685.5 \\
\hline L2-20 & GKPILFFRLK & $\mathrm{BC} 07$ & 810.5 & 687.5 \\
\hline L2-21 & YVADAPD & $\mathrm{BC08}$ & 810.5 & 689.5 \\
\hline GK-1 & KGVPRALMVE & BC09 & 813.5 & 689.5 \\
\hline L2-19 & fPRSGG & $\mathrm{BC} 10$ & 813.5 & 693.5 \\
\hline L3-7 & EEKQRIILG & BC11 & 813.5 & 695.5 \\
\hline L3-16 & KASGPAGPA & $\mathrm{BC} 12$ & 816.5 & 695.5 \\
\hline KK1-1 & RIKFFSAQTK & BC13 & 816.5 & 697.5 \\
\hline L2-15 & LAQA $\{$ hF\}RSK & BC14 & 816.5 & 699.5 \\
\hline
\end{tabular}

1016 Table 1| Mass-barcoded substrates for multiplexed urinalysis of protease activity.

1017 hF, Homophenylalanine; $f$, $d$-form phenylalanine. The barcodes are isotopically labeled 1018 Glufib peptides (EGVNDNEEGFFSAR) that share the same MS1 precursor mass for 
1019 reporter pooling but produce unique fragmented MS2 quantifier ions distinguishable by

1020 liquid chromatography with tandem mass spectrometry (LC-MS/MS). 